\title{
Mechanistic Studies of Improved Foam EOR Processes
}

Semi-Annual Report for the Period

March 1, 2003 - August 31, 2003

Principal Author:

William R. Rossen

Date Report Issued: March 31, 2003

Work Performed under Contract DE-FC26-01BC15318

The University of Texas at Austin Center for Petroleum and Geosystems Engineering

1 University Station, C0300

Austin, TX 78712-1061 


\section{DISCLAIMER}

This report was prepared as an account of work sponsored by an agency of the United States Government. Neither the Unites States Government nor any agency thereof, nor any of their employees, makes any warranty, express or implied, or assumes any legal liability or responsibility for the accuracy, completeness, or usefulness of any information, apparatus, product, or process disclosed, or represents that its use would not infringe privately owned rights. Reference herein to any specific commercial product, process, or service by trade name, trademark, manufacturer, or otherwise does not necessarily constitute or imply its endorsement, recommendation, or favoring by the United States Government or any agency thereof. The views and opinions of authors expressed herein do not necessarily state or reflect those of the United States Government or any agency thereof. 


\title{
Mechanistic Studies of Improved Foam EOR Processes
}

\author{
Contract No. DE-FC26-01BC15318 \\ The University of Texas at Austin \\ 1 University Station, C0300 \\ Austin, TX 78712-1061 \\ Contract Date: Sept. 1, 2001 \\ Anticipated Completion: Aug. 31, 2004
}

\begin{abstract}
The objective of this research is to widen the application of foam to enhanced oil recovery (EOR) by investigating fundamental mechanisms of foams in porous media. This research will lay the groundwork for more applied research on foams for improved sweep efficiency in miscible gas, steam and surfactant-based EOR. Task 1 investigates the pore-scale interactions between foam bubbles and polymer molecules. Task 2 examines the mechanisms of gas trapping, and interaction between gas trapping and foam effectiveness. Task 3 investigates mechanisms of foam generation in porous media.

Significant progress was made during this period on all three Tasks.

Regarding Task 1, we studied the behavior of foam made without polymer, with low-molecular-weight and high-molecular-weight polyacrylamide, and with xanthan polymer in sandpacks. Results consistently showed that polymer does not stabilize foam in porous media per se. Rather, it destabilizes foam to some extent, but may increase the viscosity of water sufficiently to increase the resistance to flow in spite of the lower intrinsic stability of the foam. This is consistent with the hypothesis the motivated our study. Results also showed that polymer shifts behavior from the high-quality foam-flow regime toward the low-quality regime, consistent with our initial hypothesis. Other aspects of the experimental results were puzzling and are discussed in the text of this report.

Research on Task 2 included building an apparatus for gas-phase tracer tests for direct measurement of trapped-gas saturation with foam. We also investigated the nature of the low-quality foam regime, which is thought to be controlled by gas trapping and mobilization. In both the studies of polymers and foam and separate studies of $\mathrm{CO}_{2}$ foam, we observed behavior that seems to be related to the low-quality regime, but shows unexpected trends: specifically, a decrease in pressure gradient with increasing liquid injection rate, at fixed gas injection rate. We find that such behavior is consistent with earlier models of foam viscosity in tubes, and a modified model for the low-quality regime can account for this behavior. It is not yet clear why this new regime appears in some cases and not in others. Simple modeling suggests that the answer may have to do with the sensitivity of gas trapping to pressure gradient.
\end{abstract}


Research on Task 3 continued to focus on foam generation at limited pressure gradient in sandpacks. We investigated the effects of permeability, surfactant concentration and liquid injection rates on foam generation. In addition, a careful review of published studies showed that repeated snap-off is not a plausible mechanism of foam generation in homogeneous porous media beyond the stage of initial drainage from a fully liquid-saturated state. Snap-off has been the focus of much research on foam generation and is incorporated into most mechanistic foam simulators. This finding should force a reconsideration of its role in foam generation and properties in porous media.

This period saw the publication of two articles in refereed journals:

Rossen, W. R., "A Critical Review of Roof Snap-Off as a Mechanism of Steady-State Foam Generation in Homogeneous Porous Media," Colloids Surfaces A: Physicochem Eng. Aspects, 225 (1-3) 1-24 (2003).

$\mathrm{Xu}, \mathrm{Q}$., and Rossen, W. R., "Effective Viscosity of Foam in Periodically Constricted Tubes," Colloids Surfaces A: Physicochem Eng. Aspects 216 (1-3), 175-194 (2003).

two more accepted for publication:

Shan, D. and Rossen, W.R., "Optimal Injection Strategies for Foam IOR," paper SPE 75180 prepared for presentation at the 2002 SPE/DOE Symposium on Improved Oil Recovery, Tulsa, OK, April 13-17; accepted by SPE Journal.

Kam, S. I., and Rossen, W. R., "A Model for Foam Generation in Homogeneous Porous Media," paper SPE 77698, accepted for SPE Journal (scheduled for Dec. 2003 issue).

one submitted to a journal:

Cox, S.J., Neethling, S., Rossen, W.R., Schleifenbaum, W., Schmidt-Wellenburg, P., and Cilliers, J.J., "A Theory of the Effective Yield Stress of Foam in Porous Media: The Motion of a Soap Film Traversing a Three-Dimensional Pore," subm. to Colloids Surfaces A: Physicochem Eng. Aspects.

and three manuscripts prepared for presentation at technical conferences:

Xu, Q., and Rossen, W. R., "Laboratory Study of Gas Trapping in Foam-Acid Diversion," SPE 84133 presented at the 2003 SPE Annual Technical Conference and Exhibition, Denver, CO, 5-8 Oct.

$\mathrm{Xu}, \mathrm{Q}$., and Rossen, W. R., "Experimental Study of Gas Injection in SurfactantAlternating-Gas Foam Process," SPE 84183 presented at the 2003 SPE Annual Technical Conference and Exhibition, Denver, CO, 5-8 Oct.

Kam, S. I., Frenier, W. W., Davies, S. N., and Rossen, W. R., "Experimental Study of High-Temperature Foam for Acid Diversion" paper SPE 82266 prepared for presentation at the SPE European Formation Damage Conference, The Hague, The Netherlands, 13-14 May 2003. 
TABLE OF CONTENTS

$\begin{array}{lr}\text { ABSTRACT } & \frac{\text { Page }}{3} \\ \text { OBJECTIVES } & 6 \\ \text { EXPERIMENTAL } & 6 \\ \text { RESULTS AND DISCUSSION } & 6 \\ \text { Task 1 } & 6 \\ \text { Task 2 } & 9 \\ \text { Task 3 } & 11 \\ \text { CONCLUSIONS } & 14 \\ \text { REFERENCES } & 15 \\ \text { FIGURES } & 17\end{array}$




\section{OBJECTIVES}

The objective of this research is to widen the application of foam to enhanced oil recovery (EOR) by investigating fundamental mechanisms of foams in porous media. This research will lay the groundwork for more applied research on foams for improved sweep efficiency in miscible gas, steam and surfactant-based EOR. Task 1 investigates the pore-scale interactions between foam bubbles and polymer molecules. Task 2 examines the mechanisms of gas trapping, and interaction between gas trapping and foam effectiveness. Task 3 investigates mechanisms of foam generation in porous media.

\section{EXPERIMENTAL}

The experimental techniques employed vary with the specific task addressed. Therefore the experimental techniques are discussed together with the Results and Discussion section on each task, below.

One common theme of many of the experiments is the use of sandpacks for the porous medium rather than the consolidated rock round in most oil reservoirs. Since this is a study of foam mechanisms, then as long as those mechanisms are reproduced, it is less important to conduct experiments in consolidated porous media. A review of the literature shows that all the fundamental mechanisms of foam are seen in both sandpacks and consolidated media: foam generation by mobilization at a critical pressure gradient (Gauglitz et al., 2002); foam collapse at a limiting capillary pressure (Khatib et al., 1988); two steady-state strong-foam flow regimes, at low foam quality and high quality (Alvarez et al., 2001). Sandpacks offer several advantages over lower-permeability consolidated cores: minimal mechanical trapping and retention of polymer molecules, which allows one to separate the effects of polymer on foam from direct alterations of the porous medium by polymer; reduced pressure gradients, which reduces the need for maintaining back-pressure in foam-generation experiments; and minimal mineralogical interactions between injected fluids and the medium. Therefore we explore the fundamental mechanisms of foam in sandpacks in many cases discussed below.

\section{RESULTS AND DISCUSSION}

\section{TASK 1: INTERACTIONS BETWEEN POLYMER AND FOAM}

This work is motivated by a hypothesis about how polymer interacts with foam in porous media. The hypothesis derives in turn from the observation that steady-state foam behavior appears to comprise two very different flow regimes, at high and low foam qualities (injected gas volume fraction) (Fig. 1) (Alvarez et al., 2001). The high-quality regime is controlled by lamella stability, while in the low-quality regime foam lamellae are relatively stable, bubble size is fixed, and behavior is controlled by gas trapping and mobilization. In the high-quality regime, water saturation $\mathrm{S}_{\mathrm{w}}$ is held nearly constant at the water saturation $\mathrm{S}_{\mathrm{w}}$ * corresponding to the "limiting capillary pressure" (Khatib et al., 1988; Rossen and Zhou, 1995). In the high-quality regime, applying Darcy's law to the aqueous phase at fixed water saturation $\mathrm{S}_{\mathrm{w}}{ }^{*}$ gives 


$$
\nabla \mathrm{p}=\mathrm{u}_{\mathrm{w}} \mu_{\mathrm{w}} /\left(\mathrm{k} \mathrm{k}_{\mathrm{rw}}\left(\mathrm{S}_{\mathrm{w}}{ }^{*}\right)\right)
$$

where $u_{w}$ is water superficial velocity, $\mu_{w}$ is aqueous-phase viscosity, $\mathrm{k}$ is permeability and $\mathrm{k}_{\mathrm{rw}}\left(\mathrm{S}_{\mathrm{w}}{ }^{*}\right)$ the relative permeability to the aqueous phase at $\mathrm{S}_{\mathrm{w}}{ }^{*}$. Our hypothesis is that polymer affects foam in the high-quality regime by (a) viscosifying the aqueous phase (increasing $\mu_{\mathrm{w}}$ ) and (b) stabilizing or destabilizing foam lamellae (reducing or increasing $\mathrm{S}_{\mathrm{w}}{ }^{*}$, respectively). One can distinguish between these effects by measuring the viscosity of the aqueous phase separately from the foam (accounting if possible for the effects of shear rate on polymer viscosity). If upon addition of polymer the pressure gradient in porous media in the high-quality regime increases more than does $\mu_{\mathrm{w}}$, then polymer stabilizes foam lamellae (reduces $\mathrm{S}_{\mathrm{w}}{ }^{*}$ and $\mathrm{k}_{\mathrm{rw}}$ ); if pressure gradient increases less than does $\mu_{\mathrm{w}}$, then polymer destabilizes the lamellae (raises $\mathrm{S}_{\mathrm{w}}{ }^{*}$ and $\mathrm{k}_{\mathrm{rw}}$ ). If measured $\nabla \mathrm{p}$ data are in the low-quality regime, then the relation between $\mathrm{k}_{\mathrm{rw}}\left(\mathrm{S}_{\mathrm{w}}\right)$ and foam stability is less direct, but one would still expect $\nabla \mathrm{p}$ to reflect water saturation and water viscosity, and one can separate the effects of polymer on each.

Our new experiments were conducted in sandpacks of permeability 6.6 and 16.6 darcy. The surfactant is a $1 \mathrm{wt} \%$ (as received) solution of Bio-Terge AS-40 (an alpha olefin sulfonate), manufactured by Stepan Chemical Co., in brine with $0.098 \mathrm{wt} \% \mathrm{Na}^{+}$, $0.045 \mathrm{wt} \% \mathrm{Ca}^{++}$. The surfactant product comes as about $40 \%$ active, so this represents about $0.4 \% \mathrm{wt} \%$ active surfactant. Back-pressure was about $600 \mathrm{psi}$ and nitrogen was the gas. In other respects, the coreflood apparatus was similar to that in earlier studies (Alvarez et al., 2001). Polymer solutions were $0.1 \mathrm{wt} \%$ of, respectively, a lowmolecular-weight polyacrylamide $(\mathrm{MW} \sim 500,000)$ or a high-molecular-weight polyacrylamide (MW 10-12,000,000), or a $0.05 \mathrm{wt} \%$ solution of xanthan polymer (Xanvis, from Kelco Oil Field Group) (MW 5-7,000,000). The viscosities of the three aqueous foam formulations with polymer were $1.2 \mathrm{cp}, 2.4 \mathrm{cp}$, and $3.9 \mathrm{cp}$, respectively. In all cases viscosity was independent of shear rate over the range measured, which was from 0.9 to from 8 to $19 \mathrm{~s}^{-1}$, depending on the formulation. All experiments were carried out at room temperature.

Fig. 2 shows the behavior of foam without polymer in a 6.6-darcy sandpack. There appears to be a high-quality regime (vertical $\nabla p$ contours) at lower liquid superficial velocity $\mathrm{U}_{\mathrm{w}}$. At higher $\mathrm{U}_{\mathrm{w}}$ (to the right in the plot) the $\nabla \mathrm{p}$ contours are not horizontal as expected in the low-quality regime (cf. Fig. 1), but show decreasing $\nabla p$ as $\mathrm{U}_{\mathrm{w}}$ increases at fixed gas superficial velocity $\mathrm{U}_{\mathrm{g}}$. Similar behavior is seen with polymer in the study of Romero et al. (2002). We discuss this aspect of the results further under Task 2. There is one other aspect of these results that is unexpected. Rather than a smooth transition from the high-quality to low-quality regimes (Fig. 1), one finds a drastic increase in $\nabla \mathrm{p}$ between them. Fig. 3 plots a transect through the $\nabla p$ data at $\mathrm{U}_{\mathrm{g}} \sim 0.3 \mathrm{~cm} / \mathrm{min}$. $\nabla \mathrm{p}$ increases gradually with $\mathrm{U}_{\mathrm{w}}$ in the two low- $\mathrm{U}_{\mathrm{w}}$ data points, as expected in the high-quality regime (cf. Fig. 1), and decreases gradually in the two data at high $\mathrm{U}_{\mathrm{w}}$. Between these pairs of data there is a drastic increase in $\nabla \mathrm{p}$, which implies an extremely shear-thickening response to increasing $U_{w}$. Normally, we would associate this sort of behavior with a foam-generation event (Gauglitz et al., 2002), and hysteresis. But we found the $\nabla \mathrm{p}$ data on both sides of the jump in $\nabla \mathrm{p}$ to be reproducible. At this point we have no explanation for this behavior. It appears in several additional data plots below. 
Figs. 4 to 7 show data for foam in a 16.6-darcy sandpack with, respectively, 0.1 $\mathrm{wt} \%$ low-MW polyacrylamide (MW 500,000); $0.1 \mathrm{wt} \%$ high-MW polyacrylamide (MW 10-12,000,000); $0.05 \mathrm{wt} \%$ xanthan (MW 5-7,000,000); and foam without polymer in the same sandpack after the polymer-foam experiments in that pack. The low-MW polyacrylamide hardly alters the viscosity of the aqueous phase $(1.2 \mathrm{cp})$; the solution of surfactant and high-MW polymer has a viscosity of $2.4 \mathrm{cp}$; the solution of surfactant plus xanthan has viscosity $3.9 \mathrm{cp}$.

Foam with the low-MW polyacrylamide in 16.6-darcy sand (Fig. 4) shows lower $\nabla \mathrm{p}$ than both foam without polymer in 6.6-Darcy sand (Fig. 2) and foam without polymer in 16.6-darcy sand after polymer had passed through the sand (Fig. 7). In comparing these plots it's important to note the different ranges of superficial velocities. One would not normally expect polymer to alter significantly the properties of a high-permeability sandpack. This then acts as a test of the effect of polymer on foam stability apart from any direct effect on $\nabla p$ from increasing water viscosity (Eq. 1) - because there is no appreciable increase in water viscosity in this case. The result suggests that polymer destabilizes foam. Fig. 4, like Fig. 2, shows a jump in $\nabla p$ between data at low $U_{w}$ and at higher $U_{w}$, with its implication of shear-thickening behavior.

With higher-MW polymer (Fig. 5), values of $\nabla p$ are comparable to those in Fig. 7; they are higher than in Fig. 2, but not by as much as one would expect from the increased viscosity of the aqueous phase $(2.4 \mathrm{cp})$ and Eq. 1. The values of $\nabla \mathrm{p}$ in Fig. 6, with xanthan polymer (3.9 $\mathrm{cp}$ viscosity for the aqueous phase) are likewise comparable to those on Fig. 7 and higher than in Fig. 2, but not by the factor one would expect given the increase in viscosity. Again, one concludes that polymer destabilizes foam (increases water saturation and $\mathrm{k}_{\mathrm{rw}}$ ) while it may increase $\nabla \mathrm{p}$ by viscosifying the aqueous phase. One sees in Fig. 5, but not Fig. 6, the jump in $\nabla$ p between low- $U_{w}$ and high- $U_{w}$ regimes seen in Figs. 2 and 4.

Polymer-free foam in the same sandpack as the polymer-foam data (Fig. 7) shows $\nabla \mathrm{p}$ values comparable to those with xanthan polymer, the last polymer tested in this sandpack. This leaves open the possibility that one or other polymer used had left a residual resistance factor in even this high-permeability sandpack. Otherwise, it means that polymer does not increase the pressure gradient with foam in these foam formulations. Research continues on these issues.

One goal of this work is to examine the effect of oil on foam both with and without polymer. We use decane as the oil, because in separate tests decane appeared to destabilize bulk foam made with our surfactant formulation more effectively than crude oils we had on hand. Fig. 8 shows the behavior of the polymer-free foam formulation with oil present in the sandpack. We follow here the procedure of Mamun et al. (2002). Oil is easily displaced from sandpacks by high pressure gradients. Therefore, to produce reproducible, fairly constant conditions with oil present, we inject oil along with the foam at a fixed volume fraction of injected fluids. In this case the injected oil volume fraction is $22 \%$. Fig. 8 shows decane does destabilize this foam in the sandpack. $\nabla p$ is lower than in any of the other plots at any permeability. Also, as expected (Alvarez et al., 2001), the destabilization of foam by oil causes the high-quality regime to extend its reach over a wider range of foam qualities. Experiments of foam with oil and polymer are continuing. 


\section{TASK 2: GAS TRAPPING}

During this period we began construction of an apparatus for measuring trapped gas saturation in porous media using gas-phase tracers. The apparatus is similar to a conventional coreflood facility, with a gas chromatograph at the outlet of the medium. The breakthrough curve of gas tracer at the outlet reflects the fraction of the gas phase in the medium that is flowing, and, by implication, the fraction that is trapped. We expect substantial results from this experimental study in the next reporting period.

Gas trapping is thought to play its most important role in the low-quality foamflow regime. Recent research has caused us to reconsider the nature of this regime. Figs. 2 to 7 show altered behavior in the low-quality regime: rather than pressure gradient $\nabla \mathrm{p}$ independent of liquid superficial velocity $U_{w}$ at fixed gas superficial velocity $U_{g}, \nabla p$ decreases upon increasing $U_{\mathrm{w}}$ at fixed $\mathrm{U}_{\mathrm{g}}$. Similar behavior is observed in some cases with $\mathrm{CO}_{2}$ foam above the critical pressure of $\mathrm{CO}_{2}$. Our surfactant formulation was $1 \mathrm{wt} \%$ Neodol ${ }^{\circledR} 25-9$ in a brine of $3 \mathrm{wt} \% \mathrm{NaCl}$ and $0.01 \mathrm{wt} \% \mathrm{CaCl}_{2}$. Neodol $25-9$ is a nonionic $\mathrm{C}_{12-15}$ alcohol ethoxylate with average 9 moles of ethylene oxide per mole of alcohol, manufactured by Shell Chemical Company

Figs. 9 and 10 show, respectively, liquid- $\mathrm{CO}_{2}$ foam (i.e., below the critical temperature of $\mathrm{CO}_{2}$ ) in a Boise sandstone core and a sandpack. Figs 11 and 12 show supercritical $\mathrm{CO}_{2}$ foam (i.e., above the critical temperature of $\mathrm{CO}_{2}$ ) in a Boise sandstone core and a sandpack. The conventional two foam-flow regimes are observed in the Boise core above the critical temperature of $\mathrm{CO}_{2}$ (Fig. 11). In the other cases, the unconventional behavior is observed. There is no one factor that determines whether one observes the unconventional behavior or not. A series of experiments at the New Mexico Petroleum Recovery Research Center were conducted above and below the critical temperature of $\mathrm{CO}_{2}$ with different surfactant formulations in a variety of porous media. Dong (2001) shows that all these experiments reflect either the conventional high- or low-quality foam regimes.

This behavior we report here, where $\nabla \mathrm{p}$ decreases at increasing $\mathrm{U}_{\mathrm{w}}$, may be unique to foam. It is remarkable that in two-phase flow, upon increasing the injection rate of the more viscous phase, pressure gradient should decrease. Also, to the extent that water injection rate affects bubble size, one would expect bubble size to decrease as water injection rate increases (Alvarez et al., 2001). A decrease in bubble size would give an increase in $\nabla \mathrm{p}$, not a decrease as shown here.

Since the low-quality regime is thought to be controlled by gas mobilization and trapping, we investigated this further to determine whether gas trapping is the root of the unconventional behavior.

Theoretical work summarized below suggests that the answer is "yes and no." The root of the trend where $\nabla p$ decreases as $U_{w}$ increases is in the foam viscosity function, not gas trapping. However, if gas trapping is sufficiently sensitive to pressure gradient, then $\nabla \mathrm{p}$ becomes independent of $\mathrm{U}_{\mathrm{w}}$ as in the conventional low-quality regime.

\section{Gas Effective Viscosity With Foam}

Rossen and Wang (1999) proposed their explanation for the low-quality regime based on a simple bundle-of-tubes model for foam in porous media. Water is a Newtonian, wetting fluid that occupies the narrower tubes. Gas occupies the wider tubes, but a yield stress prevents flow in these tubes unless the pressure gradient is sufficient to mobilize gas in the given tube. As a result, the widest tubes are occupied by gas that 
flows, the intermediate tubes by gas that is trapped, and the narrowest tubes by water. Gas is represented in this model as a Bingham plastic (Bird et al., 1960). If one assumes that the parameters of the Bingham plastic are fixed and constant in the low-quality regime, then an increase in $U_{w}$ causes water to displace some trapped gas from intermediate-size tubes, with no change in the flow of gas in the widest tubes. Thus $\nabla \mathrm{p}$ stays constant as $\mathrm{U}_{\mathrm{w}}$ increases at fixed $\mathrm{U}_{\mathrm{w}}$. As $\mathrm{U}_{\mathrm{g}}$ increases, $\nabla \mathrm{p}$ increases, but less than proportionately to $U_{g}$, because as a plastic fluid gas is shear-thinning in the tubes in which it flows, and because gas flows in more tubes at $\nabla \mathrm{p}$ increases.

The Bingham parameters for the gas in this model reflect the bubble size in the foam. The behavior of the low-quality regime ( $\nabla p$ independent of $\left.U_{w}\right)$ is observed only if these parameters are independent of liquid injection rate. Therefore Rossen and Wang reasoned that bubble size is independent of liquid and gas injection rates in the lowquality regime. Qualitative observations of bubble size by Alvarez et al. (2001) partially confirm this conjecture. A fixed bubble size is reasonable if bubble size is at about pore size. Foam generation by lamella division and snap-off both stop when bubbles reach about pore size, and gas diffusion would rapidly eliminate bubbles smaller than pores if they were formed (Rossen, 1996). Thus the low-quality regime reflects a fixed bubble size at roughly the volume of a pore.

\section{Model of de Vries and Wit}

However, even if bubble size is fixed, there is no reason why the rheological (e.g., Bingham) parameters of foam should be fixed. This can be seen in terms of two previously published models for foam. The first is the model of de Vries and Wit (1990) for two flow regimes similar to the high- and low-quality regimes. Fig. 13 shows that the model of de Vries and Wit in the low-quality regime fits the trend seen in much of our recent data: $\nabla p$ decreasing with increasing $\mathrm{U}_{\mathrm{w}}$ at fixed $\mathrm{U}_{\mathrm{g}}$. The model of de Vries and Wit is not entirely satisfactory, however. It makes unphysical assumptions about the nature of foam flow in tubes.

\section{Model of Hirasaki and Lawson}

The standard model for the effective viscosity of foam in capillaries is that of Hirasaki and Lawson (1985). Although usually cited for its shear-thinning rheology with respect to gas velocity, the model also predicts that foam viscosity at fixed bubble size depends on capillary pressure. Specifically, the drag on the Plateau borders between bubbles depends on how swollen or constricted the Plateau borders are; the lower the capillary pressure, the more swollen the Plateau borders, and the lower the effective viscosity of bubbles flowing through capillaries. If one assumes that bubbles flow through all pores in the medium, which are represented as capillaries of identical diameter, and that all water flows in the Plateau borders between bubbles, one obtains behavior shown in Fig. 14: $\nabla p$ decreases as $U_{w}$ increases at fixed $U_{g}$.

A somewhat more sophisticated approach builds on the bundle-of-tube model of Rossen and Wang (1999), where gas remains trapped in some tubes and water flows through its own set of narrow tubes. Here instead of representing the gas as a Bingham plastic we use an effective viscosity based on the model of Hirasaki and Lawson (1985), as adapted by Falls et al. (1989), with a yield stress, shear-thinning rheology with respect to gas velocity, and decreasing gas viscosity with decreasing capillary pressure. The results are shown in Fig. 15, for a bundle of tubes, with a log-normal distribution of tube 
diameters with $95 \%$ of the diameters within a factor of 3.4 of the median. The behavior of the unconventional regime is reproduced: $\nabla p$ decreases with increasing $U_{w}$ at fixed $\mathrm{U}_{\mathrm{g}}$.

However, if gas trapping is a very sensitive function of $\nabla p$, then one recovers the behavior of the conventional low-quality regime. In the context of this bundle-of-tubes model, gas trapping is a function of tube radius and yield stress of the foam (Bird et al., 1960). Thus in a bundle of tubes of nearly identical radius, gas trapping would be extremely sensitive to $\nabla \mathrm{p}$ : one would pass from nearly all gas trapped to nearly all gas flowing with a small increase in $\nabla$ p. An example is shown in Fig. 16, in which $95 \%$ off all tubes have a diameter within $2 \%$ of the median value. $\nabla p$ contours are horizontal, but behavior is also extremely shear-thinning with respect to gas velocity. Real foams are not nearly this shear-thinning.

The resolution of the paradox of the unconventional foam behavior is still unclear. It appears to depend on gas trapping, however, as a way to counterbalance the effect of water injection rate on capillary pressure and drag on bubbles.

\section{TASK 3: FOAM GENERATION}

We continue experiments examining foam generation with limited pressure gradient, following up on earlier research showing a minimum pressure gradient for foam generation and an unstable regime at intermediate pressure gradients (Gauglitz et al., 2002; Kam and Rossen, 2002). Our experiments are conducted in sandpacks; the same trends in foam behavior are observed in sandpacks as in consolidated core, but at lower pressure gradient (Khatib et al., 1988; Alvarez et al., 2001; Gauglitz et al., 2002). It is much more convenient to work in sandpacks than consolidated core, because at low pressure drop in a sandpack one does not need to apply back-pressure. Fluctuations in back-pressure are hard to completely eliminate, and they can introduce transient false pressure gradients into the apparatus, which can in turn trigger foam generation. The lack of elevated back-pressure does mean that gas compression can affect gas flow rate near the inlet at high pressure drops across the core. Gas flow rates are measured (though not controlled) by a Brooks Instruments Co. mass flow controller. Pressure drop across the core is set by a conventional pressure regulator in the gas line. All experiments reported here were conducted with Bio-Terge AS-40 surfactant.

Following the approach of Gauglitz et al. (2002), we conduct experiments with fixed liquid injection rate and fixed pressure drop across the core. In these experiments, the sandpack is initially saturated with brine. Then gas and brine are injected until steady state is achieved. Surfactant solution and gas are then injected at the same rates as brine and gas, for a sufficient period for surfactant solution to replace the brine in the sandpack at a pressure gradient too low to create foam. Then pressure drop on the gas line is raised in a series of steps, while holding liquid injection rate fixed.

Fig. 17 shows a typical result, with $1 \mathrm{wt} \%$ surfactant in a 45.5 darcy sandpack, with a fixed liquid interstitial velocity of $1.75 \mathrm{ft} /$ day and with pressure drop $\Delta p$ across the core fixed at a series of steps of increasing $\Delta p$. At very low $\nabla p$ (point "a" in Fig. 17), gas flows freely with high mobility. This is the "coarse foam" regime. At some point, however, an increase in pressure gradient leads to a decrease in gas interstitial velocity. If injection rates were fixed rather than pressure drop, one would observe foam generation at a gas interstitial velocity of about $70 \mathrm{ft} /$ day, with a corresponding jump upward in $\nabla \mathrm{p}$ 
to about $10 \mathrm{psi} / \mathrm{ft}$. With fixed pressure gradient, though, the onset of foam generation is indicated by a decrease in gas flow rate rather than a marked increase in $\nabla p$.

Gas flow rate continues to decrease until the sandpack is nearly plugged to gas flow at a pressure gradient of about $1 \mathrm{psi} / \mathrm{ft}$. Near points "b" and "c" in Fig. 17, gas flow rates fluctuate, suggesting that this intermediate regime is inherently unstable. At a pressure gradient above $8 \mathrm{psi} / \mathrm{ft}$, gas flow rate starts to increase to significant values again. This is the "strong foam" regime. The "high-quality" and "low-quality" regimes discussed above are both portions of the strong-foam regime. In the strong foam regime, gas flow rate is again steady, suggesting that this is a stable steady state.

Fig. 18 shows the same foam formulation in the same sandpack, but with a higher liquid superficial velocity, $6.98 \mathrm{ft} /$ day. Both the gas velocity and the pressure gradient are lower at the onset of foam generation than with the lower liquid injection rate. This is consistent with the model of Rossen and Gauglitz (1990) for foam generation in steady liquid-gas flow. In addition, the pressure gradient with strong foam is higher, as would be expected with a higher liquid injection rate.

In experiments in high-permeability porous media, one may not immediately recognize strong foam from the (relatively low) magnitude of the pressure gradient. Therefore it is helpful to plot effective gas relatively permeability $\mathrm{k}_{\mathrm{rg}}$ eff. In this context, "effective gas relative permeability" means gas mobility times the viscosity of gas without foam. In other words, all the mobility reduction of foam is lumped into $\mathrm{k}_{\mathrm{rg}}$ eff . Fig. 19 shows the $\mathrm{k}_{\mathrm{rg}}$ eff values derived from Figs. 17 and 18. In the coarse-foam regime, $\mathrm{k}_{\mathrm{rg}}$ eff is about 0.01 ; then it declines to below 0.0001 in the strong foam regime. At sufficiently high $\nabla \mathrm{p}, \mathrm{k}_{\mathrm{rg}}{ }^{\text {eff }}$ increases again, reflecting shear-thinning rheology of foam and coarsening of foam at the limiting capillary pressure (Khatib et al., 1988). There is no sharp, universal dividing point between strong foam and coarse foam, but typically $\mathrm{k}_{\mathrm{rg}}$ eff greater than 0.001 would reflect a relatively coarse foam, and $\mathrm{k}_{\mathrm{rg}}$ eff less than 0.0001 a relatively strong foam.

Figs. 20 and 21 show a similar comparison of the effect of liquid superficial velocity, in a pack of lower permeability (7.1 darcy). Liquid injection rate in Fig. 21 is near that at the higher permeability in Fig. 18. Comparison of Figs. 18 and 21 shows that reducing permeability requires a higher pressure gradient and higher gas velocity for foam generation, in agreement with the theory of Rossen and Gauglitz (1990). Comparing Figs. 20 and 21 shows again that foam is created at a lower $\nabla \mathrm{p}$ and lower gas velocity at higher liquid interstitial velocity.

Figs. 20, 22 and 23 show the effect of surfactant concentration on foam generation. At $0.1 \mathrm{wt} \%$ surfactant (Fig. 22), foam generation requires both higher velocity and higher pressure gradient than at $1 \mathrm{wt} \%$ (Fig. 20). At $0.01 \mathrm{wt} \%$ (Fig. 23), we were not able to go high enough gas rates to create a reasonably strong foam. Fig. 24 confirms that gas mobility is not low for any of the data in Fig. 23.

Thus, over the range of conditions tested, foam generation occurs at lower pressure gradient and lower gas velocity at (a) higher liquid injection rates, (b) lower permeability, and (c) higher surfactant concentration.

During this period we published a critique of Roof snap-off as the prime mechanism of foam generation in homogeneous porous media (Rossen, 2003). The evidence that seems to support Roof snap-off as the key mechanism in foam generation is impressive: experimental studies in constricted glass tubes, etched-glass micromodels, and glass beadpacks; theoretical calculations; overall trends in gas mobility with liquid and gas injection rates; and success in simulations based on foam generation by Roof 
snap-off. A careful review of these individual mechanisms reveals the following conclusions, however:

a. Studies of snap-off in glass tubes with a single constriction are not representative of porous media, because, in effect, the downstream pore body has infinite volume. Studies in glass micromodels show that at a given pore throat, once the downstream pore body fills with bubbles, Roof snap-off ceases. Moreover, studies in constricted tubes where gas and liquid are injected simultaneously are virtually guaranteed to produce snap-off, because virtually any liquid flow rate through the throat in the experiment would be greater than that observed in a corresponding throat in a porous medium.

b. The most careful study of foam in etched-glass micromodels, that of Chambers and Radke (1990), concludes that Roof snap-off (called "constriction snap-off" there) is not an important mechanism of foam generation once the downstream pore body fills with bubbles.

c. The mechanism proposed by which snap-off produces foam generation in beadpacks cannot in fact explain the experimental observations of Ransohoff and Radke (1988), cited as evidence for the role of Roof snap-off in foam generation.

d. The theoretical calculations for snap-off rates in pore constrictions based on Roof snap-off derive from initial or boundary conditions that do not apply in porous media. Without these unphysical initial or boundary conditions, snapoff does not occur in these models.

e. The commonly observed trends of gas mobility with injection rates of gas and liquid are better explained as a result of effects of foam coalescence rather than foam generation. Moreover, the trends cited in support of Roof snap-off occur only in the high-quality regime. If steady-state foam mobility were a direct reflection of foam generation by snap-off, then one could not explain the existence of the low-quality regime.

f. Similarly, the success of population-balance simulators based on foam generation by Roof snap-off is better explained by their accounting for foam coalescence at the limiting capillary pressure, which controls foam behavior at steady state. The same steady-state behavior is produced by models that do not incorporate snap-off but do incorporate the limiting capillary pressure. Therefore, the steady-state behavior does not uniquely confirm snap-off as the foam-generation mechanism.

Details of these lines of argument, and their implications, are in Rossen (2003). 


\section{CONCLUSIONS}

Detailed conclusions are listed under each task in the section on Results and Discussion above. Important overall conclusions include the following:

1. Laboartory esults so far obtained indicate that polymer does not stabilize foam in porous media per se. Rather, it destabilizes foam to some extent. Polymer may viscosify the water phase sufficiently to more-than offset the decrease in foam stability and give a higher pressure gradient with foam.

2. In a number of our experimental studies we find not the expected low-quality foam-flow regime, but a regime in which $\nabla \mathrm{p}$ decreases with increasing liquid injection rate at fixed gas injection rate. One can explain this behavior based on the decreasing viscosity of foam at fixed bubble size with decreasing capillary pressure. Gas trapping may play a role in whether one observes the conventional low-quality regime or this new regime.

3. New experiments on foam generation show foam generation occurs at lower pressure gradient and lower gas velocity at higher liquid injection rates, lower permeability, or higher surfactant concentration. Each result is consistent with earlier theory of foam generation.

4. A detailed analysis of the evidence for Roof snap-off as a prime mechanism of foam generation in homogeneous porous media concludes that all of this evidence is either based on assumptions not valid for flow in porous media or is explained better by other mechanisms. 


\section{REFERENCES}

Alvarez, J. M., Rivas, H., and Rossen, W. R.: "A Unified Model for Steady-State Foam Behavior at High and Low Foam Qualities," SPE J (Sept. 2001), 325-333.

Bird, R. B., Stewart, W. E., and Lightfoot, E. N.: Transport Phenomena, 1st ed., Wiley, New York, 1960.

Chambers, K. T., and Radke, C. J.: "Capillary Phenomena in Foam Flow Through Porous Media," in Interfacial Phenomena in Oil Recovery, N. R. Morrow, (ed.), Marcel Dekker, New York, 1990.

De Vries, A. S. and Wit, K.: "Rheology of Gas/Water Foam in the Quality Range Relevant to Steam Foam," SPERE (May 1990), 185-192.

Dong, Y.: "Experimental Study of $\mathrm{CO}_{2}$ Foam Flow in Porous Media and Application of Fractional-Flow Method to Foam Flow," MS thesis, The University of Texas at Austin, 2001.

Falls, A. H., Musters, J. J., and Ratulowski, J.: "The Apparent Viscosity of Foams in Homogeneous Bead Packs," SPERE. (May. 1989) 155.

Gauglitz, P.A., Friedmann, F., Kam, S. I, and Rossen, W.R., "Foam Generation in Homogeneous Porous Media," Chem. Eng. Sci. 57, 4037-4052 (2002).

Hirasaki, G.J. and Lawson J.B.: "Mechanisms of Foam Flow in Porous Media: Apparent Viscosity in Smooth Capillaries," SPE J (April 1985), 176-190.

Kam, S. I., and Rossen, W. R., "A Model for Foam Generation in Homogeneous Porous Media," paper SPE 77698, presented at the SPE 2002 Annual Technical Conference and Exhibition, San Antonio, 29 Sept. - 2 Oct; accepted for publication in SPEJ.

Khatib, Z.I., Hirasaki, G.J., and Falls, "Effects of Capillary Pressure on Coalescence and Phase Mobilities in Foam Flowing Through Porous Media," SPERE (Aug. 1988), 919-926.

Ransohoff, T. C., and Radke, C. J.: "Mechanisms of Foam Generation in Glass-Bead Packs," SPERE (May 1988), 573-585.

Romero, C., Alvarez, J. M., and Muller, A. J.: "Micromodel Studies of PolymerEnhanced Foam Flow Through Porous Media," paper SPE 75179 presented at the 2002 SPE/DOE Improved Oil Recovery Symposium, Tulsa, OK, April 13-17.

Rossen, W. R., "A Critical Review of Roof Snap-Off as a Mechanism of Steady-State Foam Generation in Homogeneous Porous Media," Colloids Surfaces A: Physicochem Eng. Aspects, 225 1-24 (2003). 
Rossen, W.R., "Foams in Enhanced Oil Recovery," in R. K. Prud'homme and S. Khan, ed., Foams: Theory, Measurements and Applications, Marcel Dekker, New York, 1996, pp. 413-464.

Rossen, W.R. and Wang, M.-W., "Modeling Foams for Acid Diversion," SPE Journal (June 1999), 92-100.

Rossen, W.R. and Gauglitz, P.A., "Percolation Theory of Creation and Mobilization of Foams in Porous Media," AIChE J. 36, 1176-1188 (1990).

Rossen, W.R. and Zhou, Z.H.: "Modeling Foam Mobility at the Limiting Capillary Pressure," SPE Adv. Tech. 3, 146 (1995). 


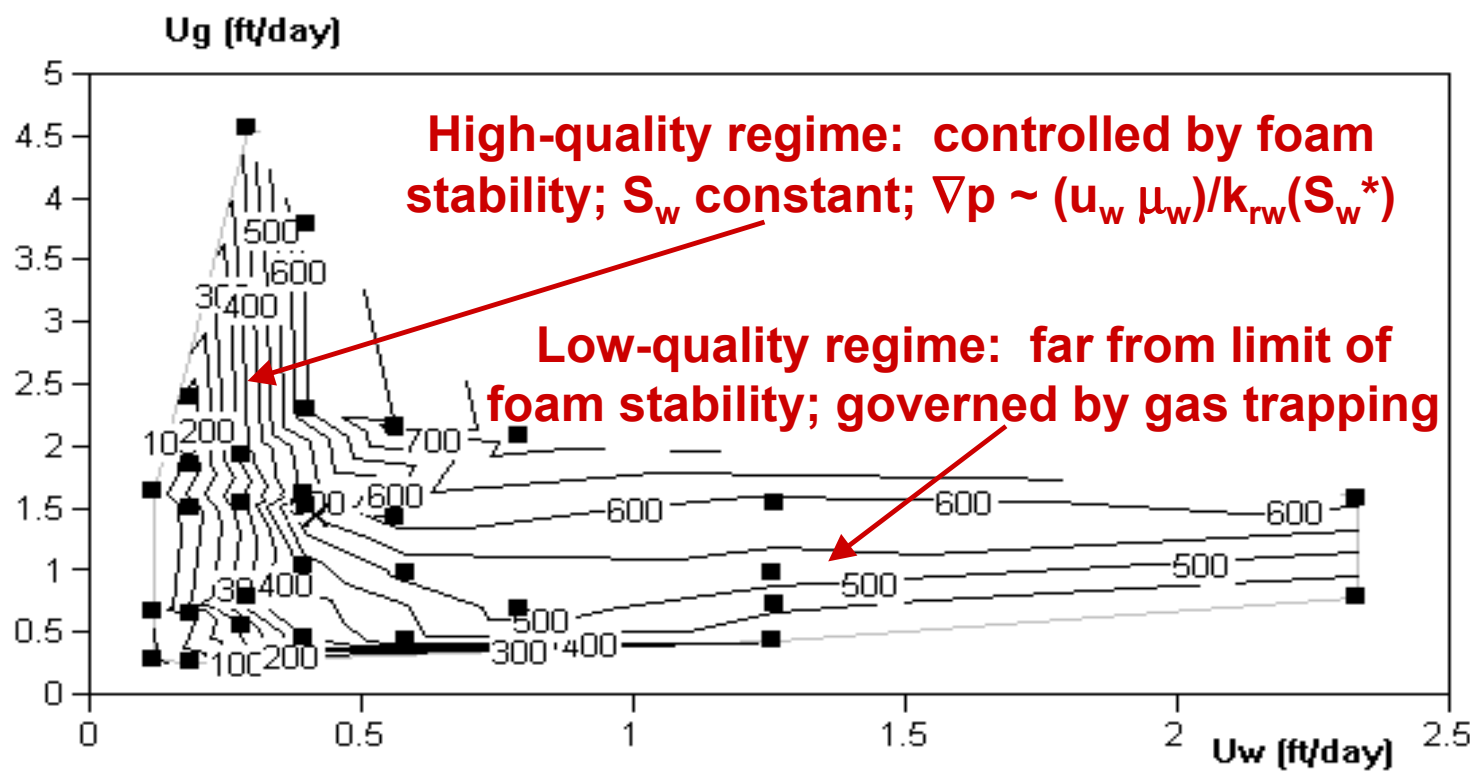

Fig. 1. Steady-state pressure gradient as a function of superficial velocities of gas $\left(\mathrm{U}_{\mathrm{g}}\right)$ and water $\left(\mathrm{U}_{\mathrm{w}}\right)$ for one $\mathrm{N}_{2}$ foam formulation in a Berea core, from Alvarez et al. (2001), illustrating the two conventional steady-state strong- foam regimes. Dark dots represent individual data.

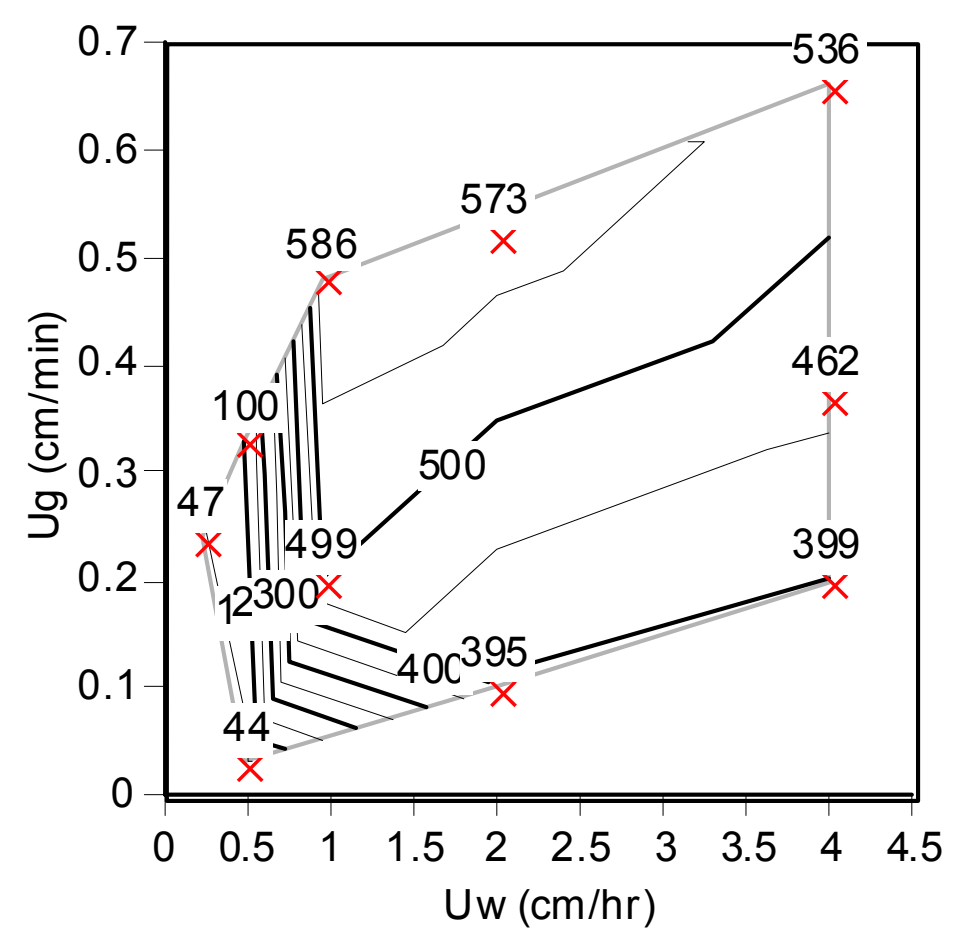

Fig. 2. Steady-state pressure gradient as a function of superficial velocities of gas $\left(U_{g}\right)$ and water $\left(\mathrm{U}_{\mathrm{w}}\right)$ for $\mathrm{N}_{2}$ foam without polymer in a 6.6-darcy sandpack. X symbols represent individual data, and the numbers above them the measured values of pressure gradient. 


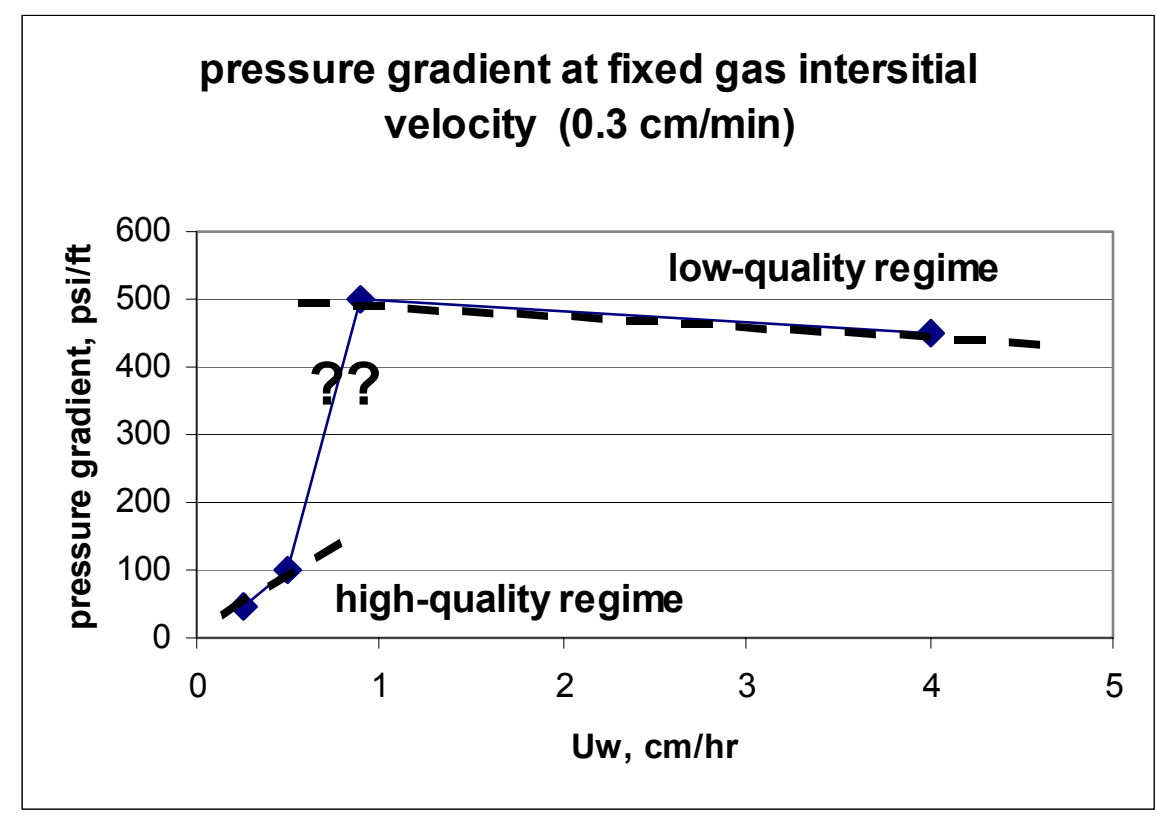

Fig. 3. Approximate transect through $\nabla \mathrm{p}$ data of Fig. 2 at $\mathrm{U}_{\mathrm{g}}=0.3 \mathrm{~cm} / \mathrm{min}$, showing apparent abrupt jump between high-quality regime and low-quality regime.

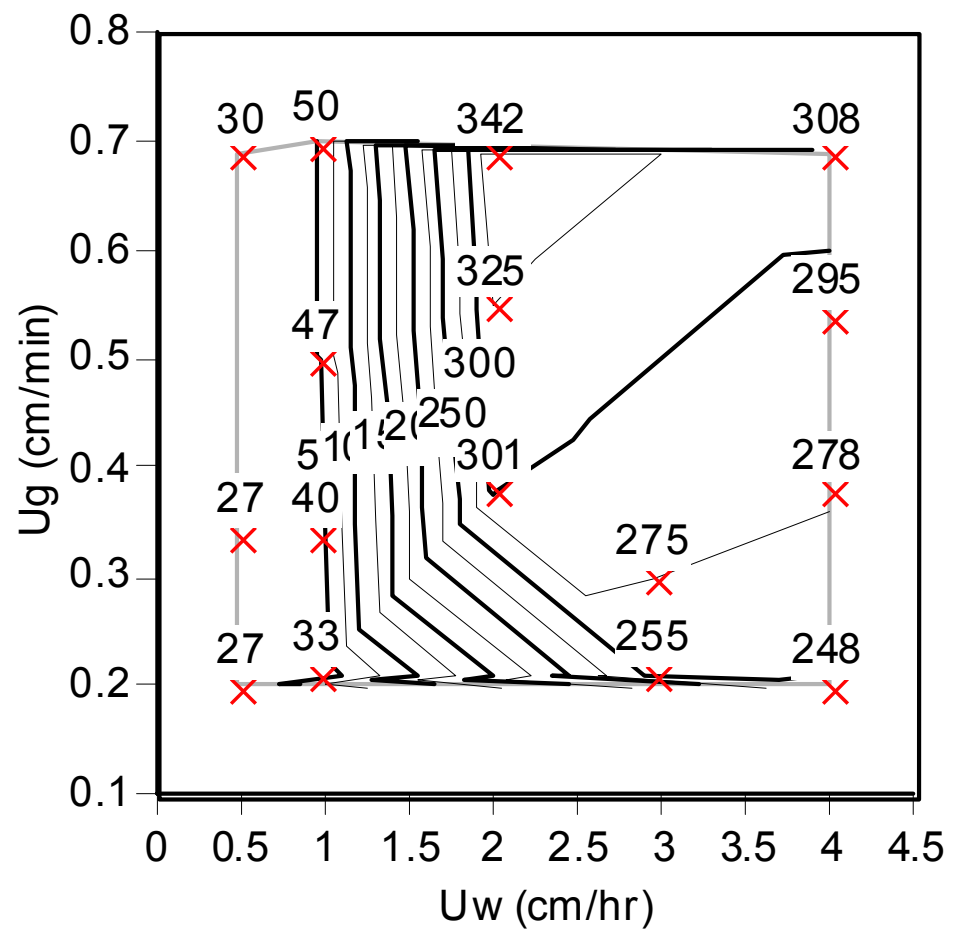

Fig. 4. Steady-state pressure gradient as a function of superficial velocities of gas $\left(\mathrm{U}_{\mathrm{g}}\right)$ and water $\left(\mathrm{U}_{\mathrm{w}}\right)$ for $\mathrm{N}_{2}$ foam with $0.1 \mathrm{wt} \%$ low-MW polyacrylamide polymer in a 16.6-darcy sandpack. X symbols represent individual data, and the numbers above them the measured values of pressure gradient. 


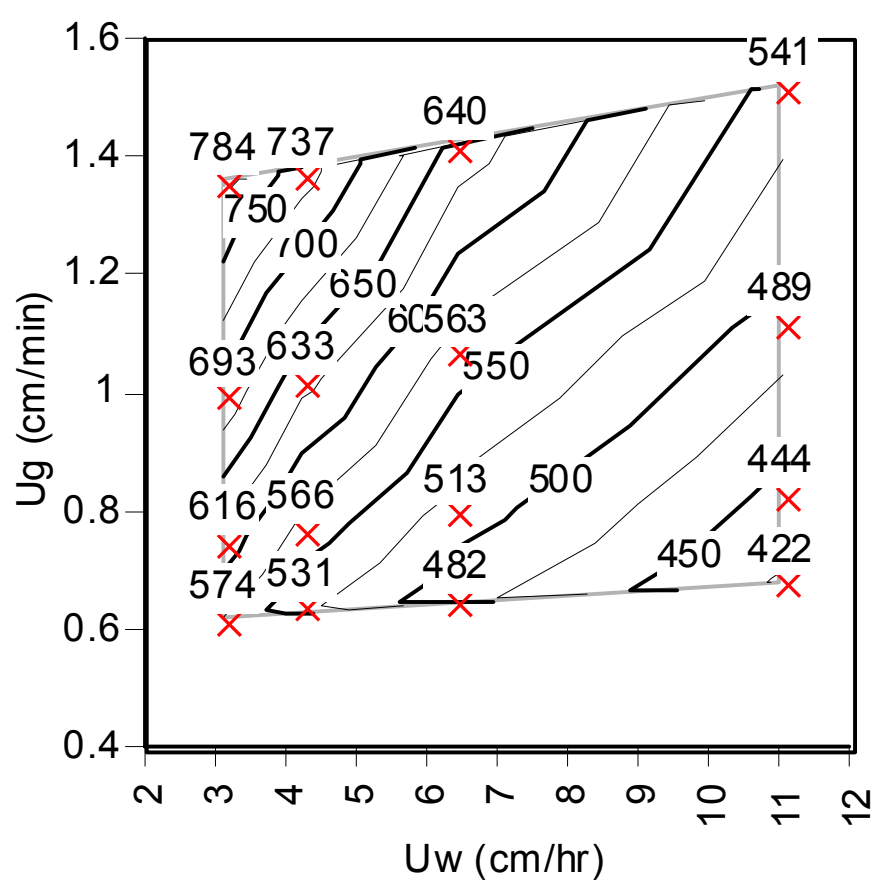

Fig. 5. Steady-state pressure gradient as a function of superficial velocities of gas $\left(\mathrm{U}_{\mathrm{g}}\right)$ and water $\left(\mathrm{U}_{\mathrm{w}}\right)$ for $\mathrm{N}_{2}$ foam with $0.1 \mathrm{wt} \%$ high-MW polyacrylamide polymer in a 16.6-darcy sandpack. $\mathrm{X}$ symbols represent individual data, and the numbers above them the measured values of pressure gradient.

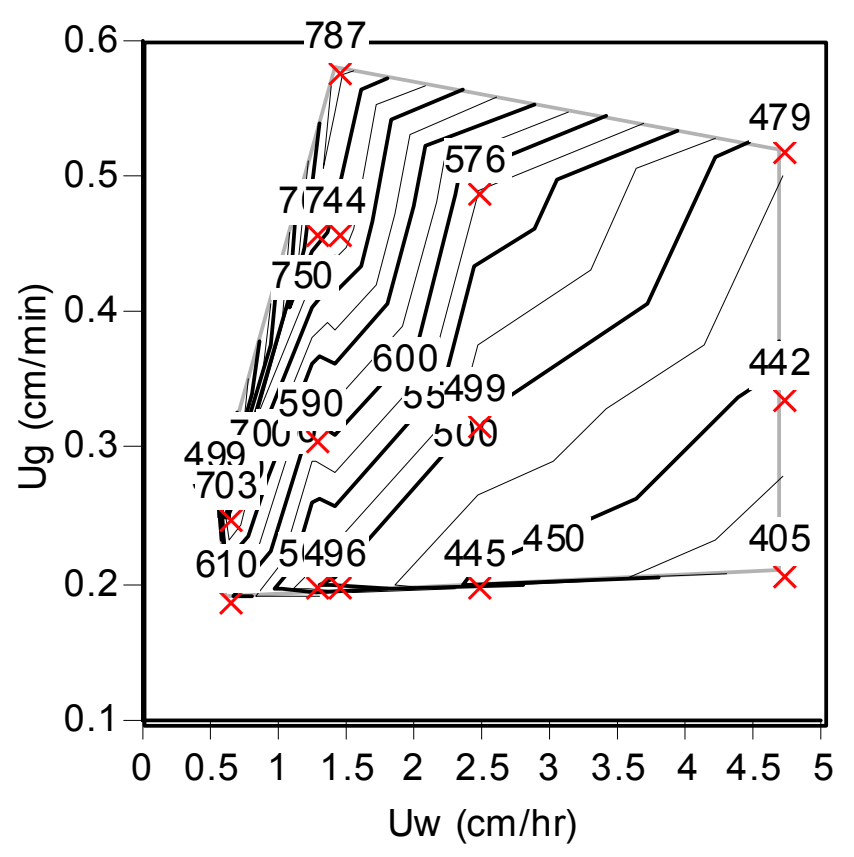

Fig. 6. Steady-state pressure gradient as a function of superficial velocities of gas $\left(\mathrm{U}_{\mathrm{g}}\right)$ and water $\left(\mathrm{U}_{\mathrm{w}}\right)$ for $\mathrm{N}_{2}$ foam with $0.05 \mathrm{wt} \%$ xanthan polymer in a 16.6-darcy sandpack. X symbols represent individual data, and the numbers above them the measured values of pressure gradient. 


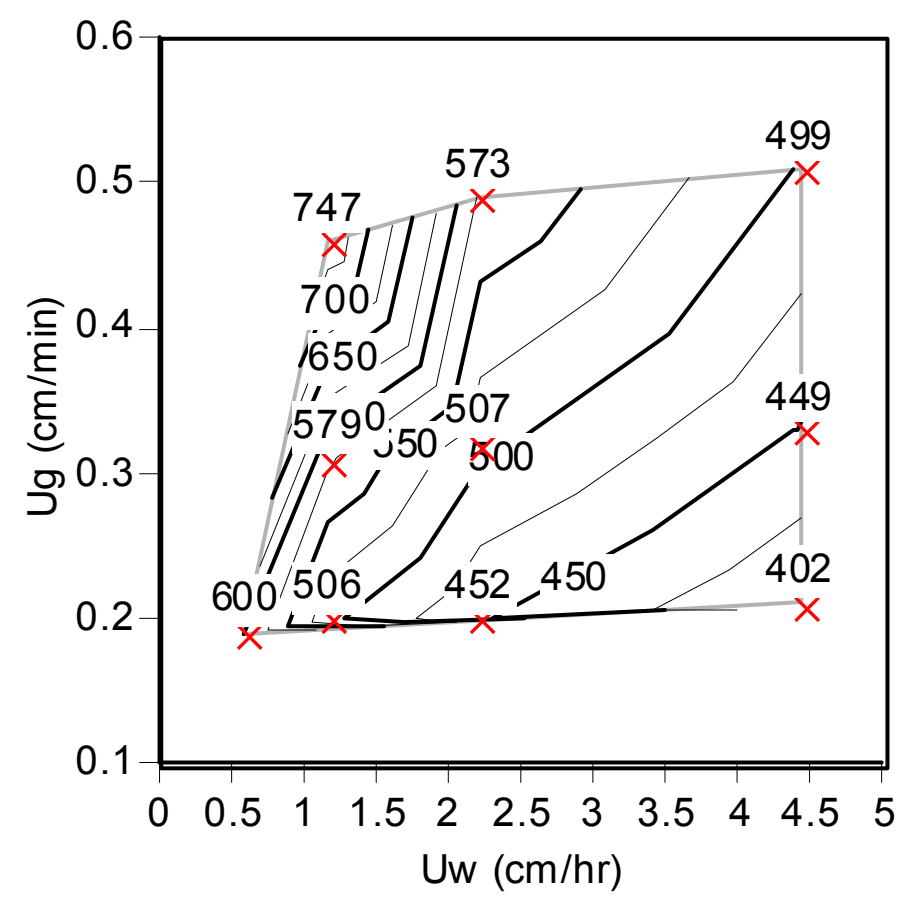

Fig. 7. Steady-state pressure gradient as a function of superficial velocities of gas $\left(\mathrm{U}_{\mathrm{g}}\right)$ and water $\left(\mathrm{U}_{\mathrm{w}}\right)$ for $\mathrm{N}_{2}$ foam without polymer in a 16.6-darcy sandpack, into which polymer foam had previously been injected. X symbols represent individual data, and the numbers above them the measured values of pressure gradient.

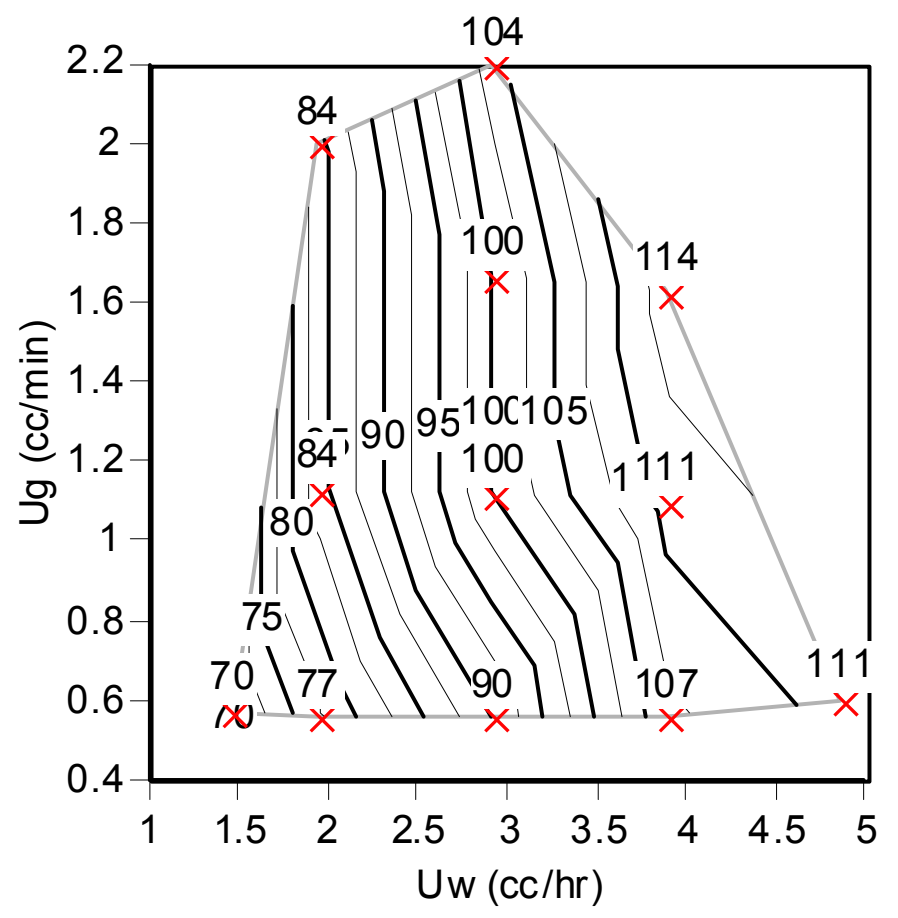

Fig. 8. Steady-state pressure gradient as a function of superficial velocities of gas $\left(\mathrm{U}_{\mathrm{g}}\right)$ and water $\left(\mathrm{U}_{\mathrm{w}}\right)$ for $\mathrm{N}_{2}$ foam without polymer in a 16.6-darcy sandpack, with decane injected simultaneously with the foam. $\mathrm{X}$ symbols represent individual data, and the numbers above them the measured values of pressure gradient. 


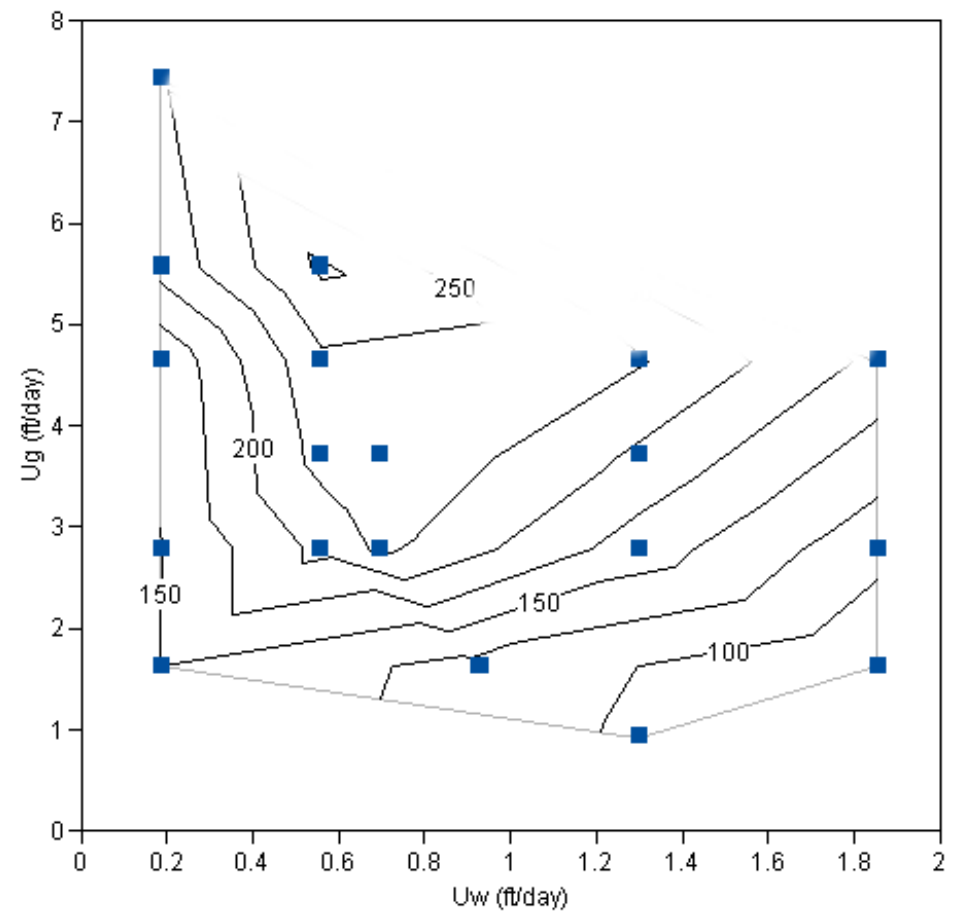

Fig. 9. Steady-state pressure gradient as a function of superficial velocities of gas $\left(\mathrm{U}_{\mathrm{g}}\right)$ and water $\left(\mathrm{U}_{\mathrm{w}}\right)$ for $\mathrm{CO}_{2}$ foam at 2000 psi and room temperature in a Boise sandstone core. Dark dots represent individual data.

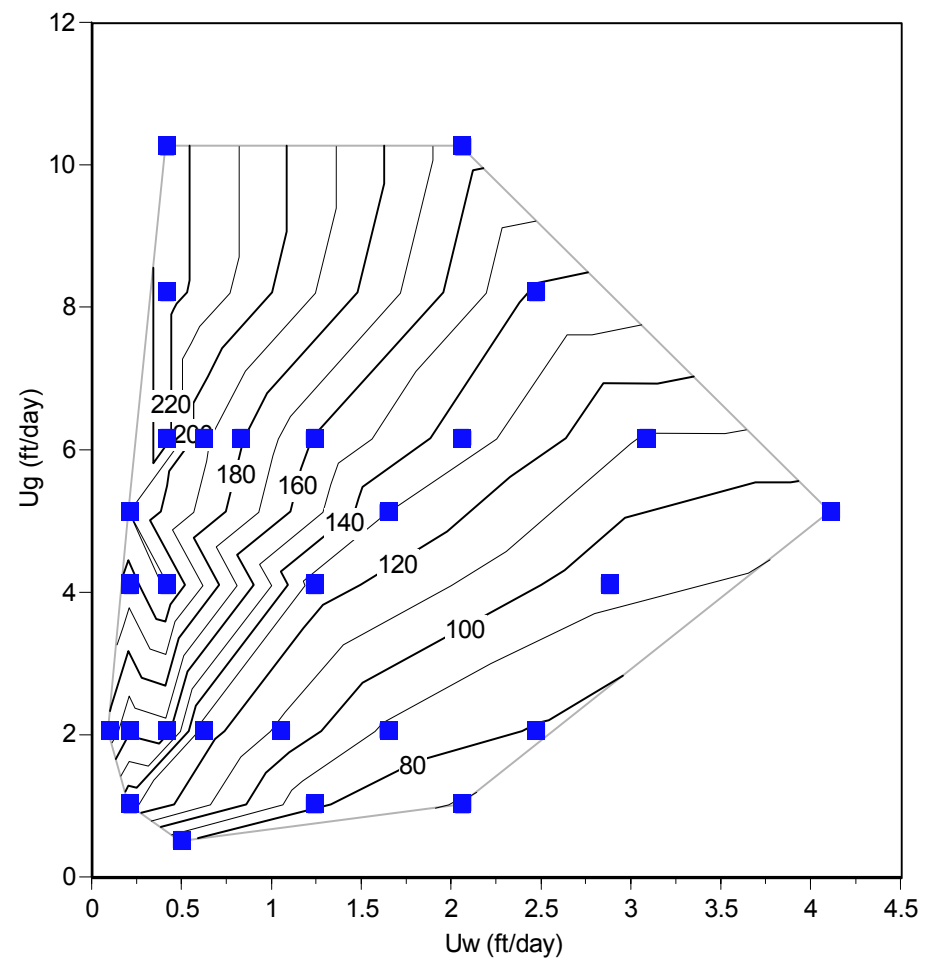

Fig. 10. Steady-state pressure gradient as a function of superficial velocities of gas $\left(\mathrm{U}_{\mathrm{g}}\right)$ and water $\left(\mathrm{U}_{\mathrm{w}}\right)$ for $\mathrm{CO}_{2}$ foam at 2000 psi and room temperature in a sandpack. Dark dots represent individual data. 


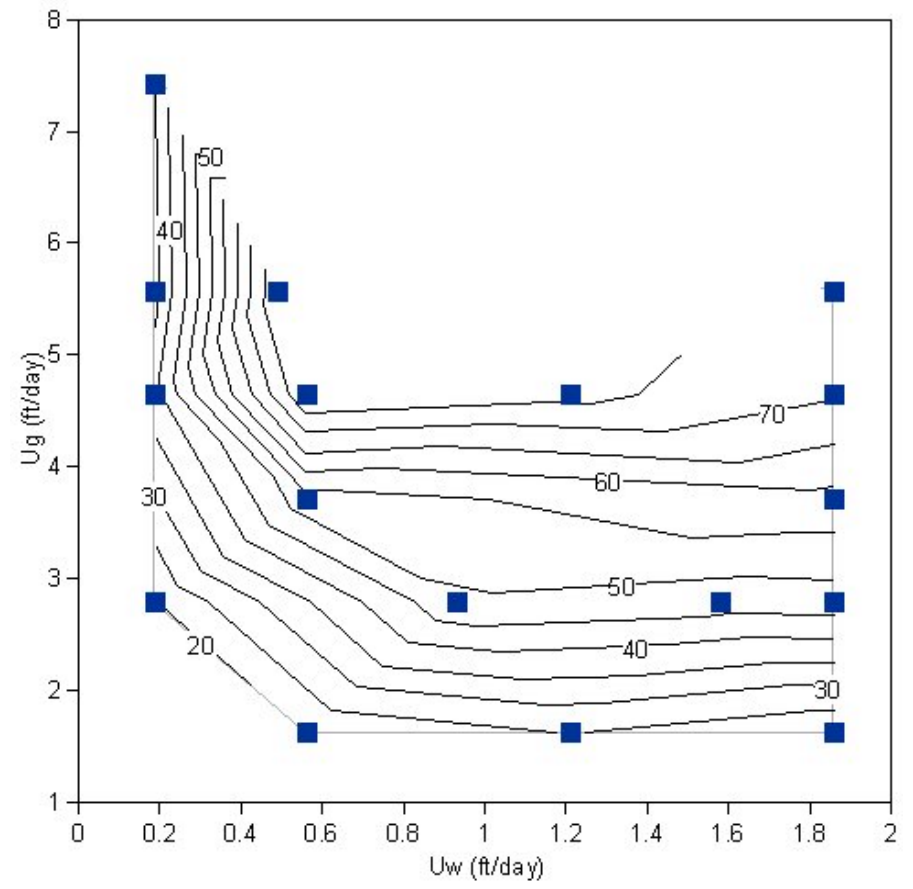

Fig. 11. Steady-state pressure gradient as a function of superficial velocities of gas $\left(\mathrm{U}_{\mathrm{g}}\right)$ and water $\left(\mathrm{U}_{\mathrm{w}}\right)$ for $\mathrm{CO}_{2}$ foam at $2000 \mathrm{psi}$ above the critical temperature of $\mathrm{CO}_{2}$ in a Boise sandstone core. Dark dots represent individual data.

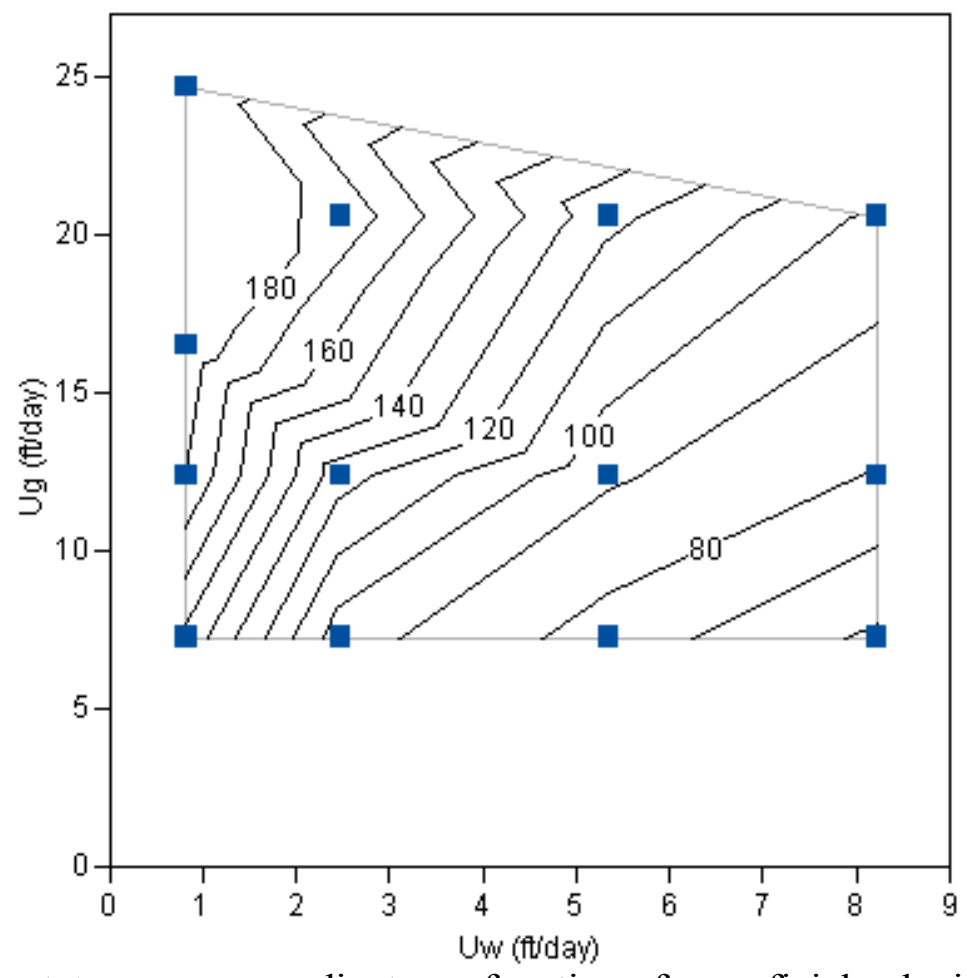

Fig. 12. Steady-state pressure gradient as a function of superficial velocities of gas $\left(\mathrm{U}_{\mathrm{g}}\right)$ and water $\left(\mathrm{U}_{\mathrm{w}}\right)$ for $\mathrm{CO}_{2}$ foam at $2000 \mathrm{psi}$ above the critical temperature of $\mathrm{CO}_{2}$ in a sandpack. Dark dots represent individual data. 


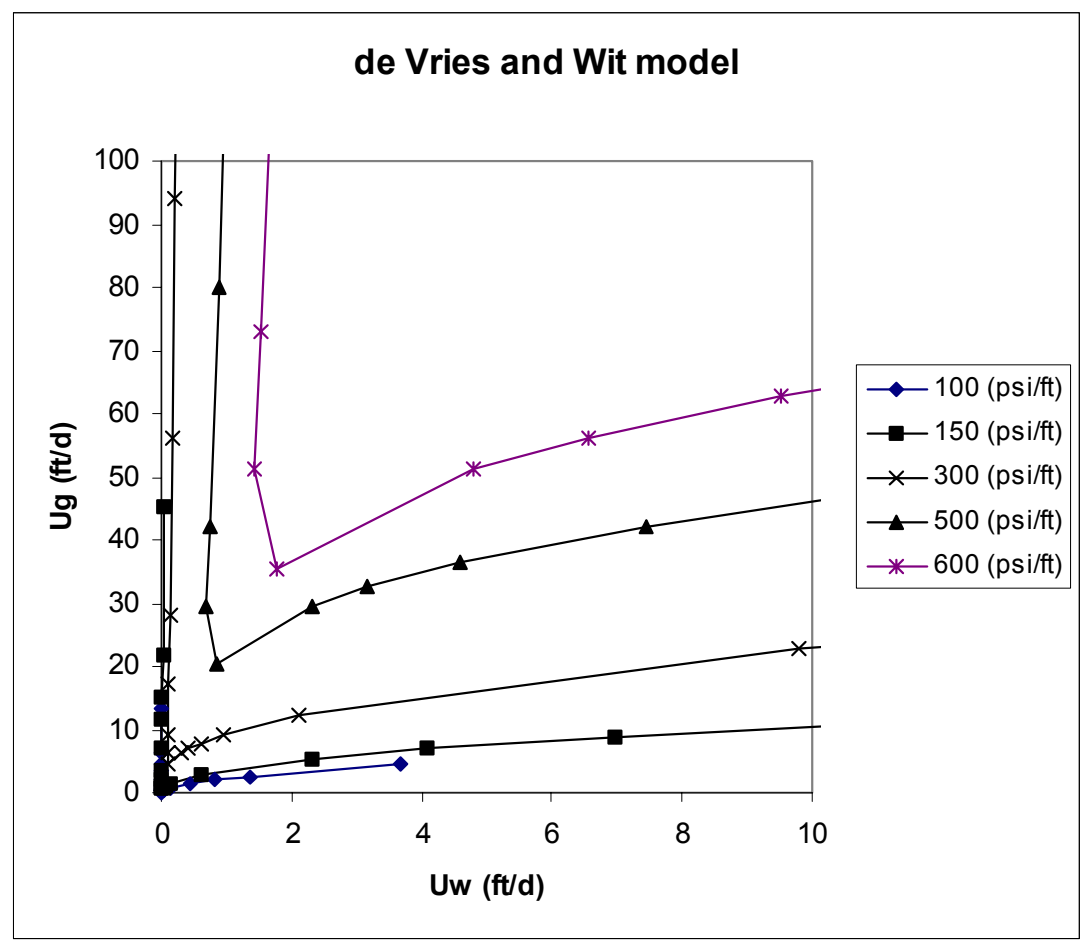

Fig. 13. Steady-state pressure gradient as a function of superficial velocities of gas $\left(U_{g}\right)$ and water $\left(\mathrm{U}_{\mathrm{w}}\right)$ for foam based on model of de Vries and Wit (1990). Symbols represent model-calculated values, not data.

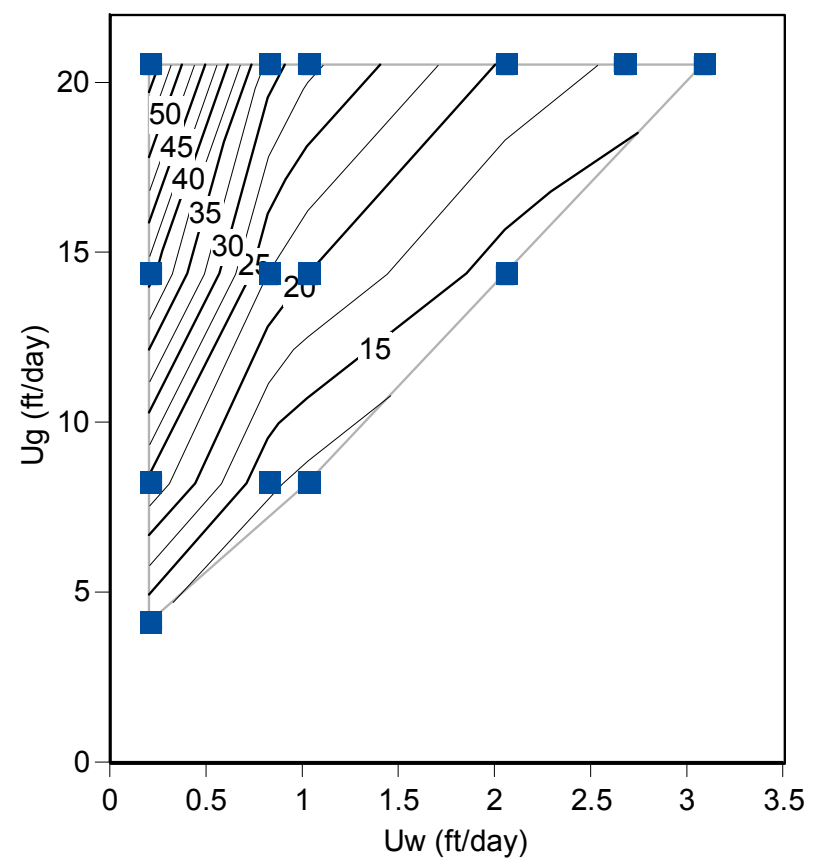

Fig. 14. Steady-state pressure gradient as a function of superficial velocities of gas $\left(U_{g}\right)$ and water $\left(U_{w}\right)$ for foam in the low-quality regime, based on model of Hirasaki and Lawson (1985), assuming foam flows through tubes of identical diameter, and that all water flows as Plateau borders between bubbles. Dark squares represent model-calculated values, not data. 


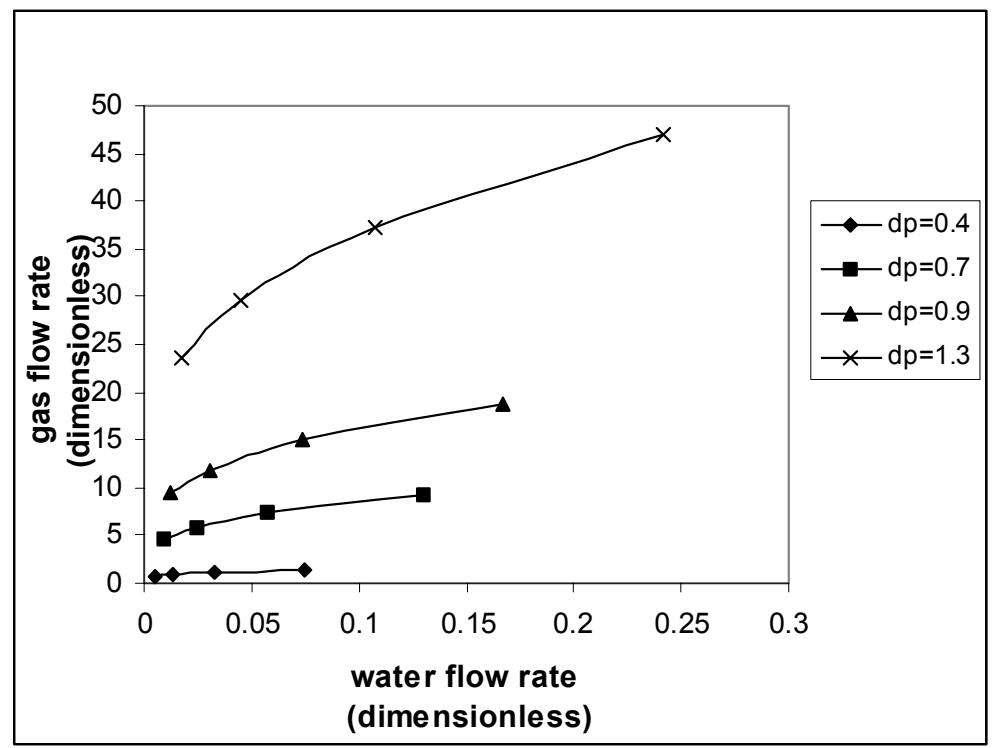

Fig. 15. Steady-state pressure gradient as a function of superficial velocities of gas $\left(U_{g}\right)$ and water $\left(\mathrm{U}_{\mathrm{w}}\right)$ for foam in the low-quality regime, based on combining the rheological models of Falls et al. (1989) and Hirasaki and Lawson (1985) with bundle-of-tubes model for foam of Rossen and Wang (1999). In this case 95\% of the capillaries have a diameter within a factor of 3.4 of the mean. Symbols represent model-calculated values, not data.

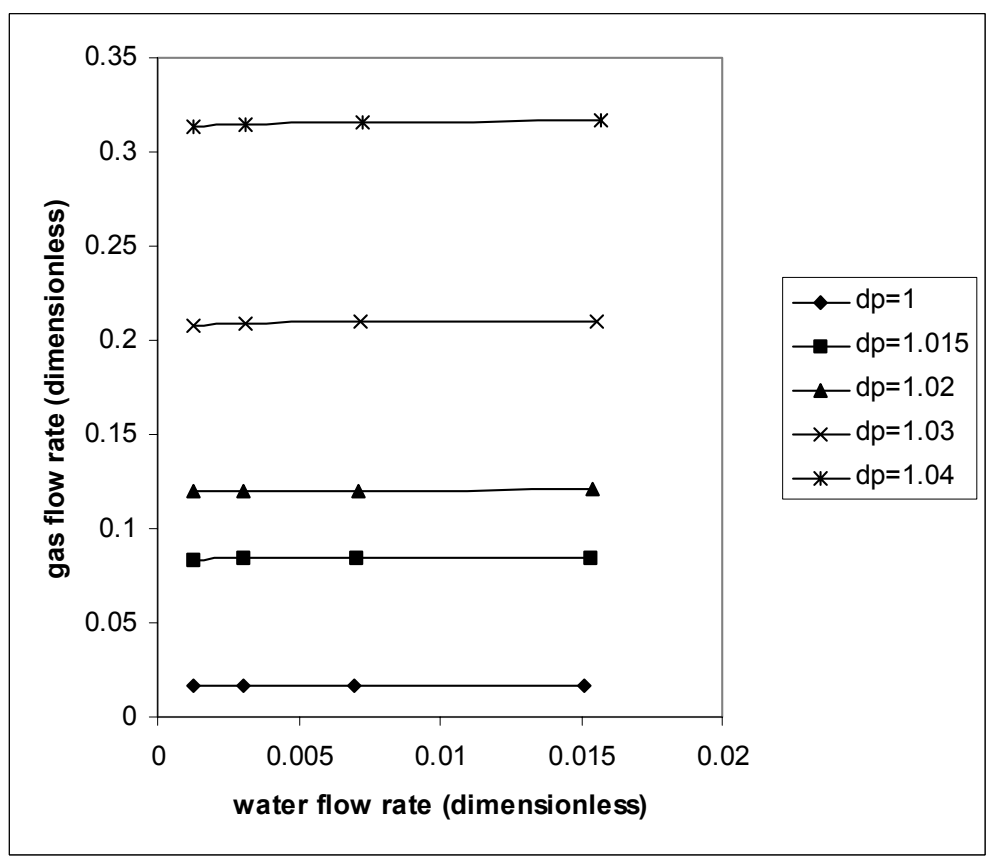

Fig. 16. Steady-state pressure gradient as a function of superficial velocities of gas $\left(U_{g}\right)$ and water $\left(\mathrm{U}_{\mathrm{w}}\right)$ for foam in the low-quality regime, based on combining the rheological models of Falls et al. (1989) and Hirasaki and Lawson (1985) with bundle-of-tubes model for foam of Rossen and Wang (1999). In this case 95\% of the capillaries have a diameter within a factor of 1.02 of the mean. Symbols represent model-calculated values, not data. 


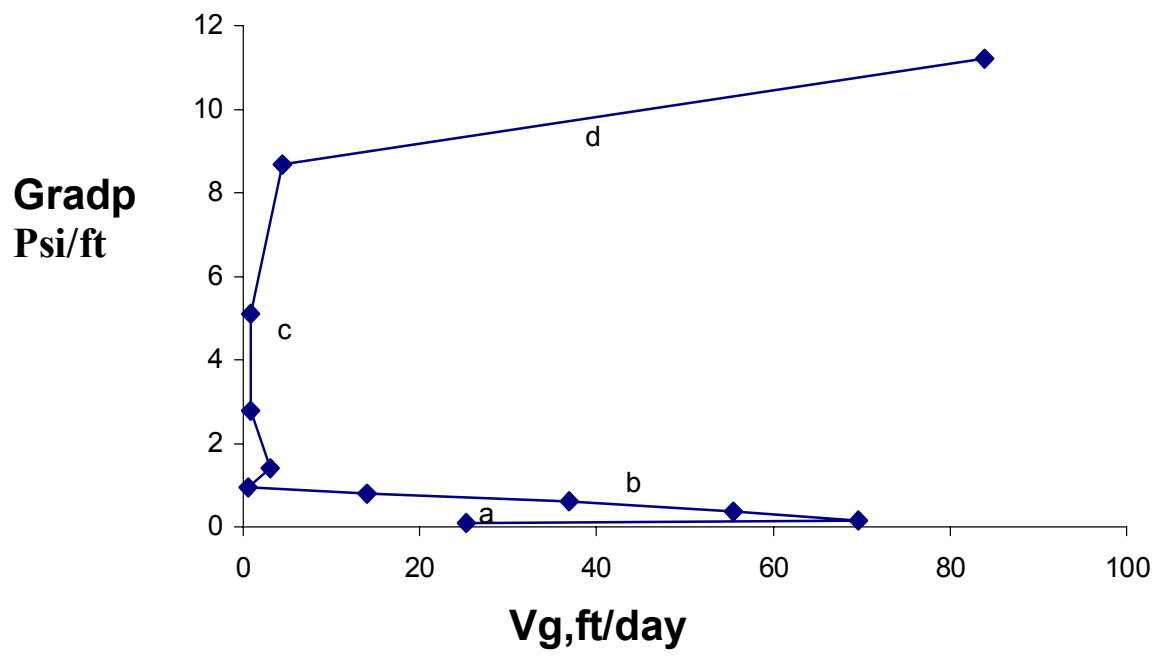

Fig. 17. Pressure gradient as a function of gas interstitial velocity $\mathrm{V}_{\mathrm{g}}$ in a 45.5-darcy sandpack; $1 \mathrm{wt} \%$ surfactant; liquid interstitial velocity $\mathrm{V}_{\mathrm{w}}=1.745 \mathrm{ft} /$ day.

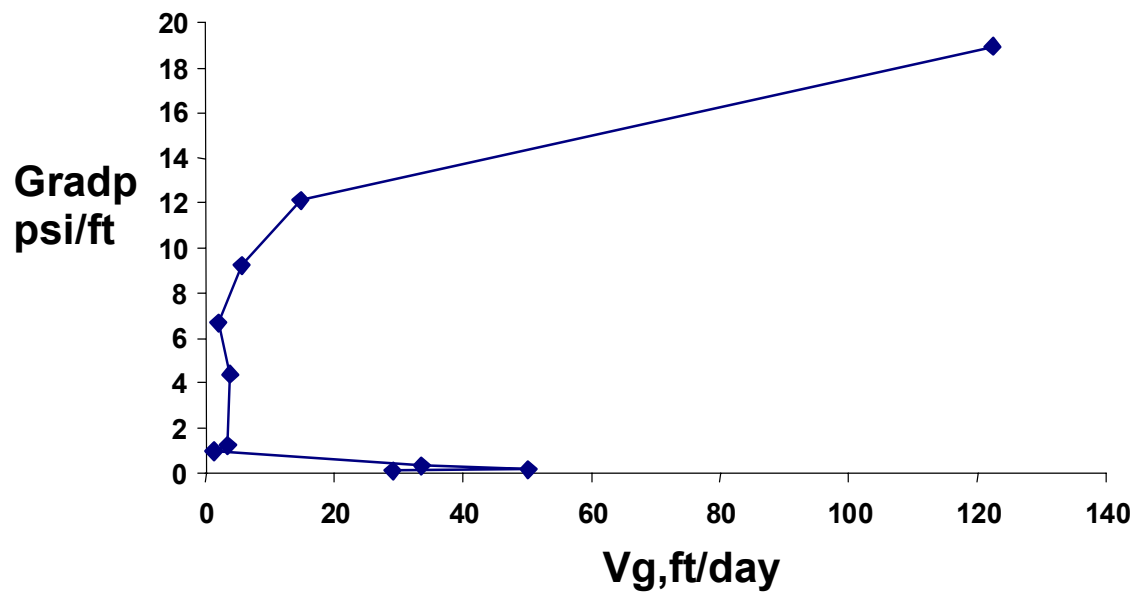

Fig. 18. Pressure gradient as a function of gas interstitial velocity $\mathrm{V}_{\mathrm{g}}$ in a 45.5-darcy sandpack; $1 \mathrm{wt} \%$ surfactant; liquid interstitial velocity $\mathrm{V}_{\mathrm{w}}=6.98 \mathrm{ft} / \mathrm{day}$. 


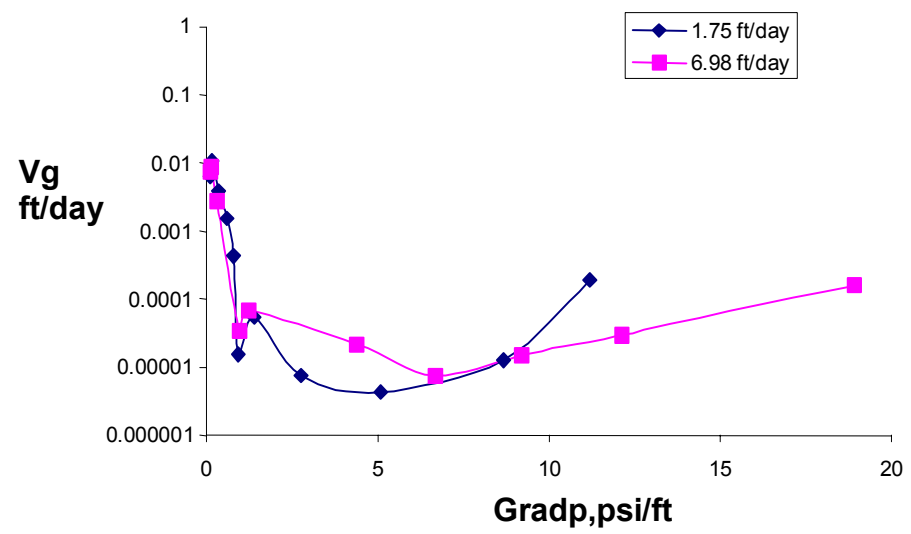

Fig. 19. Effective gas relative permeability (incorporating entire effect of foam on gas mobility into gas relative permeability) for experiments in Figs. 17 and 18.

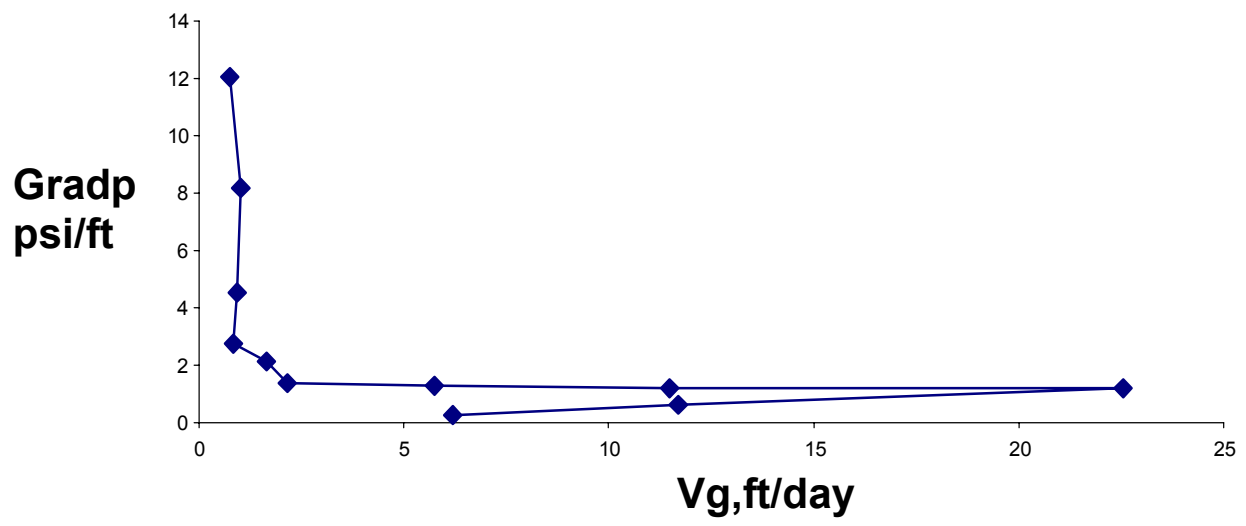

Fig. 20. Pressure gradient as a function of gas interstitial velocity $\mathrm{V}_{\mathrm{g}}$ in a 7.1-darcy sandpack; $1 \mathrm{wt} \%$ surfactant; liquid interstitial velocity $\mathrm{V}_{\mathrm{w}}=13.6 \mathrm{ft} /$ day. 


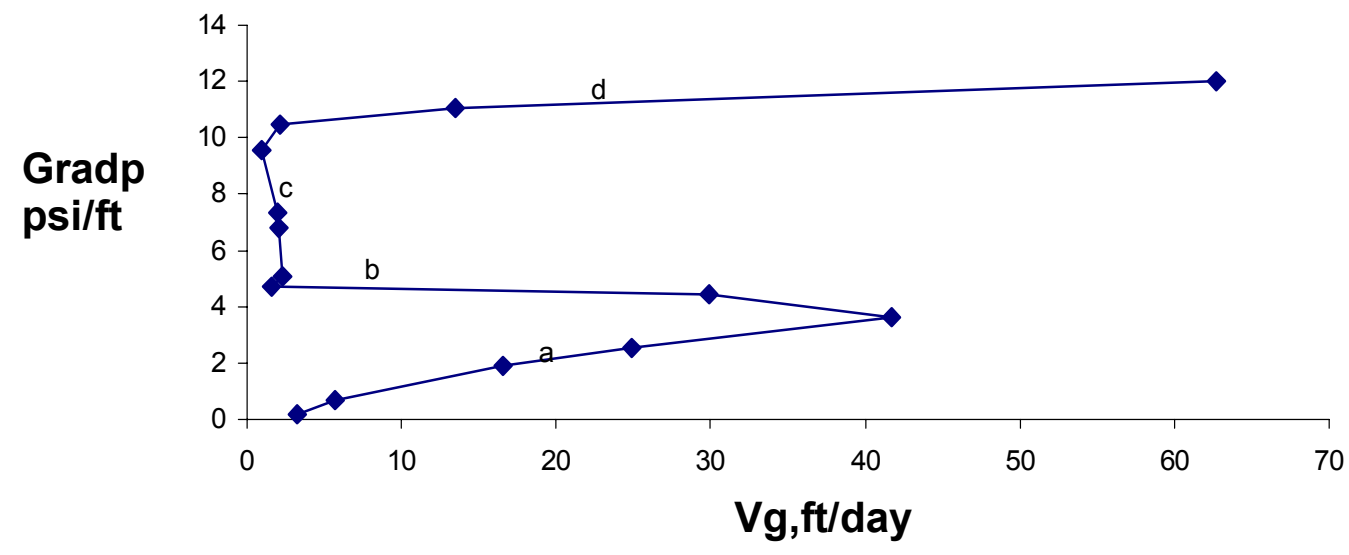

Fig. 21. Pressure gradient as a function of gas interstitial velocity $V_{g}$ in a 7.1-darcy sandpack; $1 \mathrm{wt} \%$ surfactant; liquid interstitial velocity $\mathrm{V}_{\mathrm{w}}=13.6 \mathrm{ft} / \mathrm{day}$.

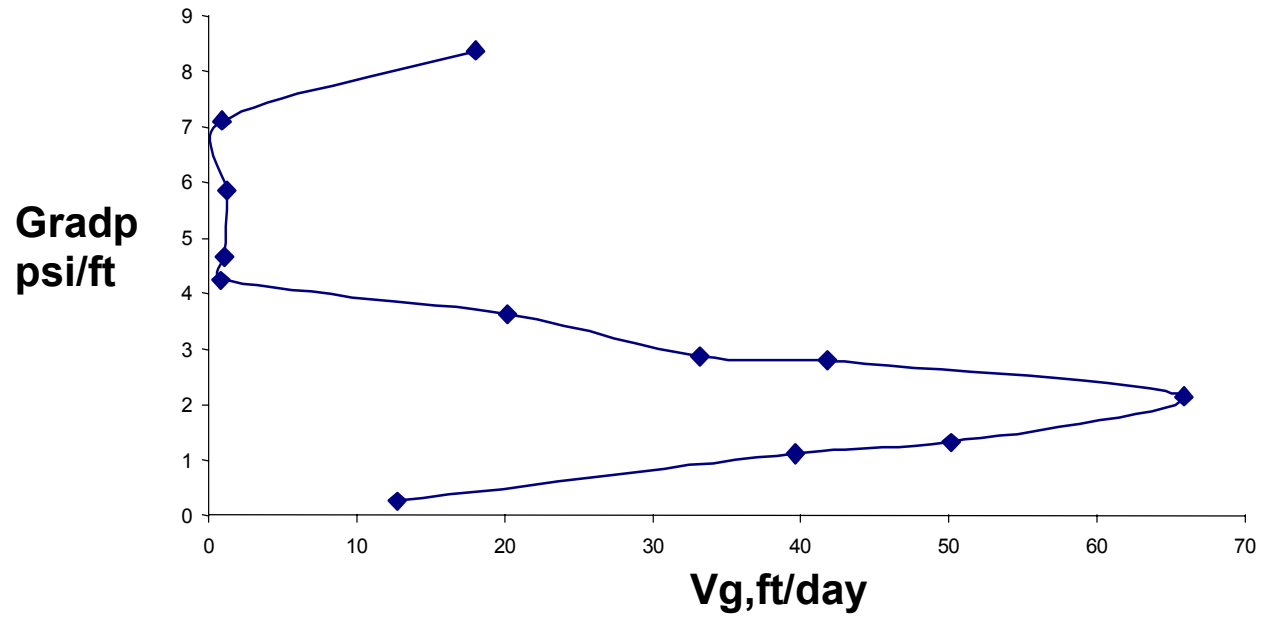

Fig. 22. Pressure gradient as a function of gas interstitial velocity $\mathrm{V}_{\mathrm{g}}$ in a 7.1-darcy sandpack; $0.1 \mathrm{wt} \%$ surfactant; liquid interstitial velocity $\mathrm{V}_{\mathrm{w}}=13.6 \mathrm{ft} / \mathrm{day}$. 


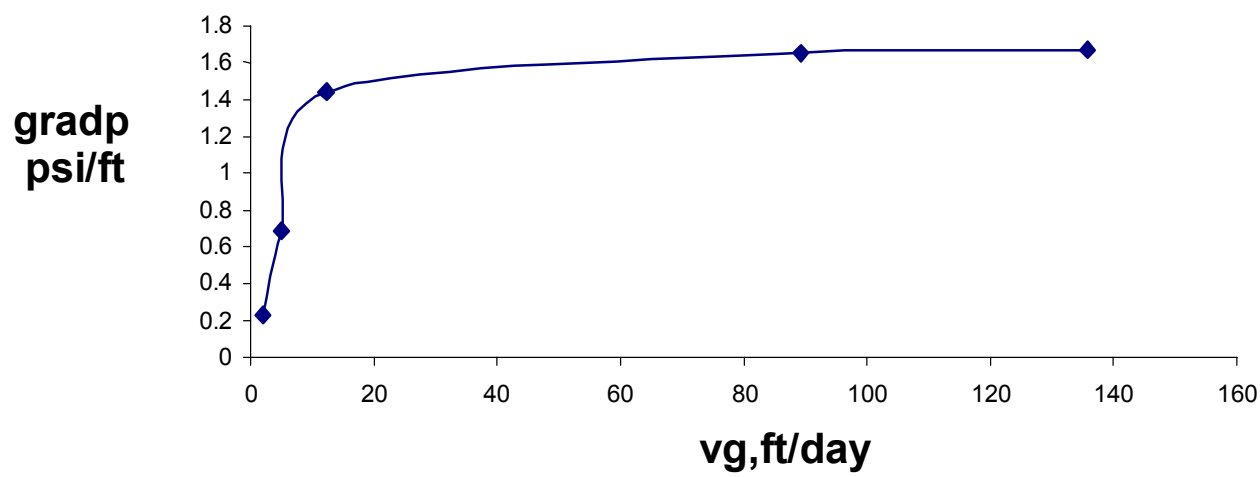

Fig. 23. Pressure gradient as a function of gas interstitial velocity $V_{g}$ in a 7.1-darcy sandpack; $0.01 \mathrm{wt} \%$ surfactant; liquid interstitial velocity $\mathrm{V}_{\mathrm{w}}=13.6 \mathrm{ft} / \mathrm{day}$.

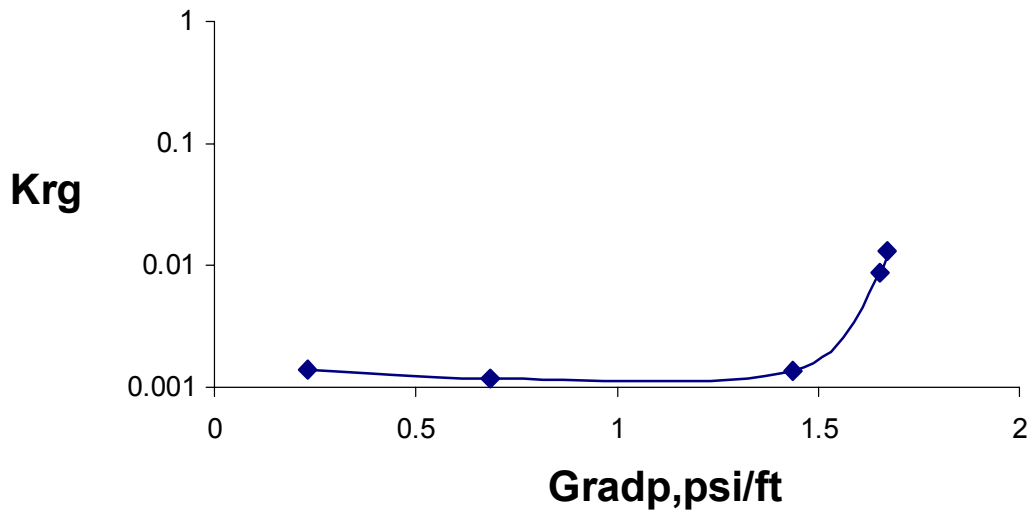

Fig. 24. Effective gas relative permeability (incorporating entire effect of foam on gas mobility into gas relative permeability) for experiments in Figs. 17 and 18. 